\title{
Fixed Point Results in Orthogonal Neutrosophic Metric Spaces
}

\author{
Umar Ishtiaq, ${ }^{1}$ Khalil Javed $\mathbb{D},{ }^{1}$ Fahim Uddin, ${ }^{1,2}$ Manuel de la Sen $\mathbb{D}^{3},{ }^{3}$ Khalil Ahmed, \\ and Muhammad Usman Ali iD ${ }^{4}$ \\ ${ }^{1}$ Department of Mathematics \& Statistics, International Islamic University Islamabad, Islamabad, Pakistan \\ ${ }^{2}$ NUIST Reading Academy, 219 Ningliu Road, Nanjing, Jiangsu 210044, China \\ ${ }^{3}$ Institute of Research and Development of Processes, Faculty of Science and Technology, \\ Univeraity of the Basque Country Campus of Leioa (Bizkaia), 644-Leioa, Spain \\ ${ }^{4}$ Department of Mathematics, COMSATS University Islamabad, Attock Campus, Attock, Pakistan
}

Correspondence should be addressed to Khalil Javed; khalil.msma551@iiu.edu.pk

Received 8 May 2021; Revised 12 July 2021; Accepted 28 July 2021; Published 9 August 2021

Academic Editor: Jesus M. Munoz-Pacheco

Copyright (C) 2021 Umar Ishtiaq et al. This is an open access article distributed under the Creative Commons Attribution License, which permits unrestricted use, distribution, and reproduction in any medium, provided the original work is properly cited.

Neutrosophy deals with neutrosophic logic, probability, and sets. Actually, the neutrosophic set is a generalization of the classical set, fuzzy set, and intuitionistic fuzzy set. A neutrosophic set is a mathematical notion serving issues containing inconsistent, indeterminate, and imprecise data. The notion of intuitionistic fuzzy metric space is useful in modelling some phenomena, where it is necessary to study the relationship between two probability functions. In this study, the concept of an orthogonal neutrosophic metric space is initiated. It is a generalization of the neutrosophic metric space. Some fixed point results are investigated in this setting. For the validity of the obtained results, some nontrivial examples are given.

\section{Introduction}

Zadeh [1] was the first to put forward the concept of fuzzy sets (FSs), and this idea has deeply influenced many scientific fields since its inception. Using the concepts of probabilistic metric space and fuzzy sets, fuzzy metric space (FMS) was introduced in [2]. Kaleva and Seikkala [3] defined the FMS as a distance between two points to be nonnegative fuzzy numbers. Afterward, the utility of FMS appeared in applied sciences such as fixed point theory, image and signal processing, medical imaging, and decision-making. This classical result was generalized in different spaces, and different structures were attained using this topic, and one may recall the existing notions, ordered theoretic results in fuzzy $b$ metric spaces [4], fuzzy b-metric-like spaces [5], orthogonal control fuzzy metric spaces [6], fuzzy generalized prioritized weighted average operator [7], fuzzy C-means clustering with locality preservation [8], the measure of divergence with fuzzy [9], and many more.
In 1986, Zadeh [1] introduced the concept of intuitionistic fuzzy sets (IFSs), which is considered as a new version of the idea of the classical set, defined by Hezarjaribi [10]. Park [11] utilized the idea of IFSs and initiated the concept of intuitionistic fuzzy metric spaces (IFMSs). Later, Kirișci and Simsek [12] introduced the notion of neutrosophic metric spaces (NMSs) which deal with membership, nonmembership, and naturalness functions. Simsek and Kirişci [13] and Sowndrarajan et al. [14] proved some fixed point results in the setting of NMSs.

On the other hand, Eshaghi et al. [15] introduced the approach of an orthogonal set (OS) and generalized the Banach fixed point (FP) theorem. Furthermore, fixed point results on orthogonal (generalized) metric spaces have been provided by Senapati et al. [16], Javed et al. [17], and Uddin et al. [18]. Using the fuzzy structure, Hezarjaribi [10] initiated the notion of an orthogonal fuzzy metric space (OFMS) and established the Banach contraction principle. 
In this study, we have introduced the notion of orthogonal neutrosophic metric space, which is a generalization of FMS, IFMS, and NMS, and we have provided nontrivial examples to justify our claim that our results would be more efficient in dealing with many scientific problems than those in the literature.

The main objectives of this study are as follows.

(1) To introduce the concept of orthogonal neutrosophic metric space (ONMSs)

(2) To prove some fixed point results in the sense of orthogonal neutrosophic metric space

(3) To enhance existing literature of fuzzy metric spaces and fuzzy fixed point theory

This study is organized with some rudimentary concepts of IFMS, NMS, OSs, and O-sequences. The concept of ONMSs is discussed in detail, and some fixed point results with nontrivial examples are imparted. A conclusion is provided for the obtained results.

In the end, some basic definitions which are important for the understanding of this study and proofs of some new results (Theorem 2 and Corollary 1) are accommodated in the Appendix section to avoid obscurity and vagueness.

Throughout this study, CTN is used for a continuous triangular norm and CTCN for a continuous triangular conorm.

\section{Preliminaries}

In this section, some basic definitions are given that are helpful to understand the main results.

Park introduced the concept of intuitionistic fuzzy metric spaces (IFMSs) and utilized this idea to investigate fixed point results. Park defined the notion of IFMSs as follows.

Definition 1 (See [11]). Suppose $E \neq \varnothing$ is an arbitrary set, assume a five-tuple $(E, R, S, *, \Delta)$, where $*$ is a CTN, $\Delta$ is a $\mathrm{CTCN}$, and $R, S$ are the FSs on $E \times E \times(0, \infty)$. If $(E, R, S, *, \Delta)$ meet the following circumstances for all $\beta, \delta, \partial \in E$ and $\pi, \lambda>0$,

(B1) $R(\beta, \delta, \lambda)+S(\beta, \delta, \lambda) \leq 1$

(B2) $R(\beta, \delta, \lambda)>0$

(B3) $R(\beta, \delta, \lambda)=1 \Longleftrightarrow \beta=\delta$

(B4) $R(\beta, \delta, \lambda)=R(\delta, \beta, \lambda)$

(B5) $R(\beta, \partial,(\lambda+\pi)) \geq R(\beta, \delta, \lambda) * R(\delta, \partial, \pi)$

(B6) $R(\beta, \delta, \Delta)$ is a nondecreasing (ND) function of $\mathbb{R}^{+}$ and $\lim _{\lambda \rightarrow \infty} R(\beta, \delta, \lambda)=1$

(B7) $S(\beta, \delta, \lambda)>0$

(B8) $S(\beta, \delta, \lambda)=0 \Longleftrightarrow \beta=\delta$

(B9) $S(\beta, \delta, \lambda)=S(\delta, \beta, \lambda)$
(B10) $S(\beta, \partial,(\lambda+\pi)) \leq S(\beta, \delta, \lambda) \Longleftrightarrow S(\delta, \partial, \pi)$

(B11) $S(\beta, \delta, \Delta)$ is a nonincreasing (NI) function of $\mathbb{R}^{+}$ and $\lim _{\lambda \longrightarrow \infty} S(\beta, \delta, \lambda)=0$

Then, $(E, R, S, *, \Delta)$ is an IFMS.

The concept of neutrosophic metric spaces was discussed by Kirişci and Simsek, and they defined the said concept as follows:

Definition 2 (See [11]). Suppose $E \neq \varnothing$, assume a five-tuple $(E, R, S, T, *, \Delta)$, where $*$ is a CTN, $\Delta$ is a CTCN, and $R, S, T$ are the neutrosophic sets (NSs) on $E \times E \times(0, \infty)$. If $(E, R, S, T, *, \Delta)$ meet the following circumstances for all $\beta, \delta, \partial \in E$ and $\pi, \lambda>0$,

$$
\begin{aligned}
& \text { (N1) } R(\beta, \delta, \lambda)+S(\beta, \delta, \lambda)+T(\beta, \delta, \lambda) \leq 3 \\
& \text { (N2) } R(\beta, \delta, \lambda)>0 \\
& \text { (N3) } R(\beta, \delta, \lambda)=1 \Longleftrightarrow \beta=\delta \\
& \text { (N4) } R(\beta, \delta, \lambda)=R(\delta, \beta, \lambda) \\
& \text { (N5) } R(\beta, \partial,(\lambda+\pi)) \geq R(\beta, \delta, \lambda) * R(\delta, \partial, \pi)
\end{aligned}
$$

(N6) $R(\beta, \delta, \Delta)$ is a nondecreasing (ND) function of $\mathbb{R}^{+}$and $\lim _{\lambda \longrightarrow \infty} R(\beta, \delta, \lambda)=1$

(N7) $S(\beta, \delta, \lambda)<1$

(N8) $S(\beta, \delta, \lambda)=0 \Longleftrightarrow \beta=\delta$

(N9) $S(\beta, \delta, \lambda)=S(\delta, \beta, \lambda)$

(N10) $S(\beta, \partial,(\lambda+\pi)) \leq S(\beta, \delta, \lambda) \Delta S(\delta, \partial, \pi)$

(N11) $S(\beta, \delta, \Delta)$ is a nonincreasing (NI) function of $\mathbb{R}^{+}$ and $\lim _{\lambda \longrightarrow \infty} S(\beta, \delta, \lambda)=0$

(N12) $T(\beta, \delta, \lambda)<1$

(N13) $T(\beta, \delta, \lambda)=0 \Longleftrightarrow \beta=\delta$

(N14) $T(\beta, \delta, \lambda)=T(\delta, \beta, \lambda)$

(N15) $T(\beta, \partial,(\lambda+\pi)) \leq T(\beta, \delta, \lambda) \Delta T(\delta, \partial, \pi)$

(N16) $T(\beta, \delta, \Delta)$ is a nonincreasing (NI) function of $\mathbb{R}^{+}$ and $\lim _{\lambda \rightarrow \infty} T(\beta, \delta, \lambda)=0$

(N17) If $\lambda \leq 0$, then $R(\beta, \delta, \lambda)=0, S(\beta, \delta, \lambda)=1$ and $T(\beta, \delta, \lambda)=1$

Then, $(E, R, S, T, *, \Delta)$ is a NMS.

Eshaghi et al. introduced the concept of orthogonal sets using a binary relation and defined it as:

Definition 3 (See [15]). Assume $E \neq \Delta$ and $\perp \in E \times E$ is a binary relation. Assume there exists $\beta_{0} \in E$, such that $\beta_{0} \perp \beta$ or $\beta \perp \beta_{0}$ for all $\beta \in E$. Thus, $E$ is said to be an OS. Furthermore, we denote OS by $(E, \perp)$.

Definition 4 (See [15]). Suppose that $(E, \perp)$ is an OS. A sequence $\left\{\beta_{n}\right\}$ for $n \in \mathbb{N}$ is called an $\mathrm{O}$-sequence if $\left(\forall n ; \beta_{n} \perp \beta_{n+1}\right)$ or $\left(\forall n ; \beta_{n+1} \perp \beta_{n}\right)$.

More definitions and basic results related to these topics are available in the Appendix section at the end of this study. 


\section{Orthogonal Neutrosophic Metric Spaces}

Now, we introduce the notion of ONMSs and utilize this concept to investigate some fixed point results.

Definition 5. Let ( $E, R, S, T, *, \Delta, \perp)$ is called an ONMS if $E$ is a (nonempty) OS, where $*$ is a CTN, $\Delta$ is a CTCN, and $R, S, T$ are the NSs on $E \times E \times(0, \infty)$ meeting the following circumstances:

$B_{\perp}(1) \quad R(\beta, \delta, \lambda)+S(\beta, \delta, \lambda)+T(\beta, \delta, \lambda) \leq 3$ for all $\beta$, $\delta \in E, \lambda>0$, such that $\beta \perp \delta$ and $\delta \perp \beta$

$B_{\perp}(2) R(\beta, \delta, \lambda)>0$, for all $\beta, \delta \in E, \lambda>0$, such that $\beta \perp \delta$ and $\delta \perp \beta$

$B_{\perp}(3) R(\beta, \delta, \lambda)=1$ if and only if $\beta=\delta$, for all $\beta, \delta \in E, \lambda>0$, such that $\beta \perp \delta$ and $\delta \perp \beta$

$B_{\perp}(4) R(\beta, \delta, \lambda)=R(\delta, \beta, \lambda)$, for all $\beta, \delta \in E, \lambda>0$, such that $\beta \perp \delta$ and $\delta \perp \beta$

$B_{\perp}$ (5) $R(\beta, \partial,(\lambda+\pi)) \geq R(\beta, \delta, \lambda) * R(\delta, e, \pi)$, for all $\beta, \delta, \partial \in E, \lambda, \pi>0$, such that $\beta \perp \delta, \delta \perp \partial$ and $\beta \perp \partial$

$B_{\perp}(6) R(\beta, \delta, \Delta):(0, \infty) \longrightarrow[0,1]$ is continuous, for all $\beta, \delta \in E$, such that $\beta \perp \delta$ and $\delta \perp \beta$

$B_{\perp}$ (7) $S(\beta, \delta, \lambda)>0$, for all $\beta, \delta \in E, \lambda>0$, such that $\beta \perp \delta$ and $\delta \perp \beta$

$B_{\perp}(8) S(\beta, \delta, \lambda)=1$ if and only if $\beta=\delta$, for all $\beta, \delta \in E, \lambda>0$, such that $\beta \perp \delta$ and $\delta \perp \beta$

$B_{\perp}(9) S(\beta, \delta, \lambda)=S(\delta, \beta, \lambda)$, for all $\beta, \delta \in E, \lambda>0$, such that $\beta \perp \delta$ and $\delta \perp \beta$

$B_{\perp}(10) \quad S(\beta, \partial,(\lambda+\pi)) \leq S(\beta, \delta, \lambda) \Delta S(\delta, \partial, \pi)$, for all $\beta, \delta, \partial \in E, \lambda, \pi>0$, such that $\beta \perp \delta, \delta \perp \partial$, and $\beta \perp \partial$

$B_{\perp}(11) S(\beta, \delta, \Delta):(0, \infty) \longrightarrow[0,1]$ is continuous, for all $\beta, \delta \in E$, such that $\beta \perp \delta$ and $\delta \perp \beta$

$B_{\perp}(12) T(\beta, \delta, \lambda)>0$, for all $\beta, \delta \in E, \lambda>0$, such that $\beta \perp \delta$ and $\delta \perp \beta$

$B_{\perp}(13) T(\beta, \delta, \lambda)=1$ if and only if $\beta=\delta$, for all $\beta, \delta \in E, \lambda>0$, such that $\beta \perp \delta$ and $\delta \perp \beta$

$B_{\perp}$ (14) $T(\beta, \delta, \lambda)=T(\delta, \beta, \lambda)$, for all $\beta, \delta \in E, \lambda>0$, such that $\beta \perp \delta$ and $\delta \perp \beta$

$B_{\perp}(15) T(\beta, \partial,(\lambda+\pi)) \leq T(\beta, \delta, \lambda) \Delta T(\delta, \partial, \pi)$ for all $\beta, \delta, \partial \in E, \lambda, \pi>0$, such that $\beta \perp \delta, \delta \perp \partial$, and $\beta \perp \partial$

$B_{\perp}(16) T(\beta, \delta, \Delta):(0, \infty) \longrightarrow[0,1]$ is continuous, for all $\beta, \delta \in E$, such that $\beta \perp \delta$ and $\delta \perp \beta$

$B_{\perp}(17)$ If $\lambda \leq 0$ then $R(\beta, \delta, \lambda)=0, S(\beta, \delta, \lambda)=1$ and $T(\beta, \delta, \lambda)=1$, for all $\beta, \delta \in E, \lambda>0$, such that $\beta \perp \delta$ and $\delta \perp \beta$.

Then, $(E, R, S, T, *, \Delta, \perp)$ is called ONMS.

Remark 1. Every NMS is an ONMS, but the converse is not necessarily true.

Example 1. Let $E=[-5,5]$ and define a CTN as $a * b=a b$, CTCN as $a \Delta b=\max \{a, b\}$, and a binary relation $\perp$ by $\beta \perp \delta$ iff $\beta+\delta \geq 0$. Take

$$
R(\beta, \delta, \lambda)= \begin{cases}1, & \text { if } \beta=\delta, \\ \frac{\lambda}{\lambda+\max \{\beta, \delta\}}, & \text { if otherwise, }\end{cases}
$$$$
S(\beta, \delta, \lambda)= \begin{cases}0, & \text { if } \beta=\delta, \\ \frac{\max \{\beta, \delta\}}{\lambda+\max \{\beta, \delta\}}, & \text { if otherwise, }\end{cases}
$$

$$
T(\beta, \delta, \lambda)= \begin{cases}0, & \text { if } \beta=\delta, \\ \frac{\max \{\beta, \delta\}}{\lambda}, & \text { if otherwise, }\end{cases}
$$

for all $\beta, \delta \in E, \lambda>0$, then it is ONMS, but not an NMS.

Easy to see, for $\pi=\lambda=1, \beta=-1, \delta=-(1 / 2), \partial=-2$. (B5), (B10), and (B15) fail.

Remark 2. The above example is also true for CTN $a * b=$ $\min \{a, b\}$ and CTCN $a \Delta b=\max \{a, b\}$.

Remark 3. The above example is also ONMS if we take

$$
S(\beta, \delta, \lambda)= \begin{cases}0, & \text { if } \beta=\delta, \\ 1-\frac{\lambda}{\lambda+\max \{\beta, \delta\}}, & \text { if otherwise. }\end{cases}
$$

Definition 6. An O-sequence $\left\{\beta_{n}\right\}$ in an ONMS $(E, R, S, T, *, \Delta, \perp)$ is called to be orthogonal convergent (O-convergent) to $\beta \in E$, if

$$
\begin{array}{ll}
\lim _{n \longrightarrow \infty} R\left(\beta_{n}, \beta, \lambda\right)=1, & \forall \lambda>0, \\
\lim _{n \longrightarrow \infty} S\left(\beta_{n}, \beta, \lambda\right)=0, & \forall \lambda>0, \\
\lim _{n \longrightarrow \infty} T\left(\beta_{n}, \beta, \lambda\right)=0, & \forall \lambda>0 .
\end{array}
$$

Definition 7. An O-sequence $\left\{\beta_{n}\right\}$ in an ONMS $(E, R, S, *, \Delta, \perp)$ is named to be orthogonal Cauchy (OCauchy) if there exists $n \in \mathbb{N}$, such that

$$
\begin{aligned}
& \lim _{n \longrightarrow \infty} R\left(\beta_{n}, \beta_{n+p}, \lambda\right)=1, \\
& \lim _{n \longrightarrow \infty} S\left(\beta_{n}, \beta_{n+p}, \lambda\right)=0, \\
& \lim _{n \longrightarrow \infty} T\left(\beta_{n}, \beta_{n+p}, \lambda\right)=0, \\
& \text { for all } \lambda \geq 0, p \geq 1 .
\end{aligned}
$$

Definition 8. $\psi: E \longrightarrow E$ is $\perp$-continuous at $\beta \in E$ in an ONMS ( $E, R, S, T, *, \Delta, \perp$ ), whenever for each O-sequence $\left\{\beta_{n}\right\}$ for all $n \in \mathbb{N}$ in $E$; if $\lim _{n \longrightarrow \infty} R\left(\beta_{n}, \beta, \lambda\right)=1$, $\lim _{n \longrightarrow \infty} S\left(\beta_{n}, \beta, \lambda\right)=0$, and $\lim _{n \longrightarrow \infty} T\left(\beta_{n}, \beta, \lambda\right)=0$ for all $\lambda>0, \quad$ then $\quad \lim _{n \longrightarrow \infty} R\left(\psi \beta_{n}, \psi \beta, \lambda\right)=1, \quad \lim _{n \longrightarrow \infty} S$ 
$\left(\psi \beta_{n}, \psi \beta, \lambda\right)=0$, and $\lim _{n \longrightarrow \infty} T\left(\psi \beta_{n}, \psi \beta, \lambda\right)=0$ for all $\lambda>0$. Furthermore, $\psi$ is $\perp$-continuous on $E$ if $\psi$ is $\perp$-continuous at each $\beta \in E$. Also, $\psi$ is $\perp$-preserving if $\psi \beta \perp \psi \delta$ when $\beta \perp \delta$.

Definition 9. An ONMS ( $E, R, S, T, *, \Delta, \perp)$ is said to orthogonally complete (O-complete) if every $\mathrm{O}$-Cauchy sequence is convergent.

Remark 4. It is necessary for the limit of a convergent $\mathrm{O}$-sequence to be unique in an ONMS.

Example 2. Assume ONMS given in an Example 1 and define a sequence $\left\{\beta_{n}\right\}$ in $E$ by $\beta_{n}=1-(1 / n), \forall n \in \mathbb{N}$, such that $\left(\forall n ; \beta_{n} \perp \beta_{n+1}\right)$ or $\left(\forall n ; \beta_{n+1} \perp \beta_{n}\right)$. Define a CTN as $a * b=a b, \mathrm{CTCN}$ as $a \Delta b=\max \{a, b\}$, and a binary relation $\perp$ by $\beta \perp \delta$ iff $\beta+\delta \geq 0$. Take

$$
\begin{aligned}
\lim _{n \longrightarrow \infty} R\left(\beta_{n}, \beta, \lambda\right) & =\lim _{n \longrightarrow \infty} \begin{cases}1, & \text { if } \beta=\delta, \\
\frac{\lambda}{\lambda+\max \left\{\beta_{n}, \beta\right\}}, & \text { if otherwise }\end{cases} \\
& = \begin{cases}1, & \text { if } \beta=\delta, \\
\frac{\lambda}{\lambda+\max \{\beta, \beta\}}, & \text { if otherwise }\end{cases} \\
& =1,
\end{aligned}
$$$$
\lim _{n \longrightarrow \infty} S\left(\beta_{n}, \beta, \lambda\right)=\lim _{n \longrightarrow \infty} \begin{cases}0, & \text { if } \beta=\delta, \\ \frac{\max \left\{\beta_{n}, \beta\right\}}{\lambda+\max \left\{\beta_{n}, \beta\right\}}, & \text { if otherwise }\end{cases}
$$$$
= \begin{cases}0, & \text { if } \beta=\delta, \\ \frac{\max \{\beta, \beta\}}{\lambda+\max \{\beta, \beta\}}, & \text { if otherwise }\end{cases}
$$$$
=0 \text {, }
$$$$
\lim _{n \longrightarrow \infty} T\left(\beta_{n}, \beta, \lambda\right)=\lim _{n \longrightarrow \infty} \begin{cases}0, & \text { if } \beta=\delta, \\ \frac{\max \left\{\beta_{n}, \beta\right\}}{\lambda}, & \text { if otherwise }\end{cases}
$$$$
= \begin{cases}0, & \text { if } \beta=\delta, \\ \frac{\max \{\beta, \beta\}}{\lambda}, & \text { if otherwise }\end{cases}
$$$$
=0 \text {. }
$$

Similarly, for any convergent $\mathrm{O}$-sequence, limit will be unique in an ONMS.

Remark 5. It is necessary that a convergent O-sequence is $\mathrm{O}$-Cauchy in an ONMS.

Example 3. From proof of above Example 2 $\beta_{n}=1-(1 / n), \forall n \in \mathbb{N}$ is a convergent $\mathrm{O}$-sequence in an ONMS

$$
\begin{aligned}
\lim _{n \longrightarrow \infty} R\left(\beta_{n}, \beta_{n+p}, \lambda\right) & =\lim _{n \longrightarrow \infty} \begin{cases}1, & \text { if } \beta=\delta, \\
\frac{\lambda}{\lambda+\max \left\{\beta_{n}, \beta_{n+p}\right\}}, & \text { if otherwise }\end{cases} \\
& = \begin{cases}1, & \text { if } \beta=\delta, \\
\frac{\lambda}{\lambda+\max \{\beta, \beta\}}, & \text { if otherwise }\end{cases} \\
& =1,
\end{aligned}
$$

$$
\lim _{n \longrightarrow \infty} S\left(\beta_{n}, \beta_{n+p}, \lambda\right)=\lim _{n \rightarrow \infty} \begin{cases}0, & \text { if } \beta=\delta, \\ \frac{\max \left\{\beta_{n}, \beta_{n+p}\right\}}{\lambda+\max \left\{\beta_{n}, \beta_{n+p}\right\}}, & \text { if otherwise }\end{cases}
$$

$$
\begin{aligned}
= \begin{cases}0, & \text { if } \beta=\delta, \\
\frac{\max \{\beta, \beta\}}{\lambda+\max \{\beta, \beta\}}, & \text { if otherwise }\end{cases} \\
=0, \\
\lim _{n \rightarrow \infty} T\left(\beta_{n}, \beta_{n+p}, \lambda\right)=\lim _{n \rightarrow \infty} \begin{cases}0, & \text { if } \beta=\delta, \\
\frac{\max \left\{\beta_{n}, \beta_{n+p}\right\}}{\lambda}, & \text { if otherwise }\end{cases}
\end{aligned}
$$$$
= \begin{cases}0, & \text { if } \beta=\delta, \\ \frac{\max \{\beta, \beta\}}{\lambda}, & \text { if otherwise }\end{cases}
$$$$
=0 \text {, }
$$

for all $\lambda \geq 0, p \geq 1$. Similarly, every convergent $\mathrm{O}$-sequence is an $\mathrm{O}$-Cauchy sequence in an ONMS.

Lemma 1. If for some $v \in(0,1)$ and $\beta, \delta \in E$, 


$$
\begin{array}{ll}
R(\beta, \delta, \lambda) \geq R\left(\beta, \delta, \frac{\lambda}{v}\right), & \lambda>0, \\
S(\beta, \delta, \lambda) \leq S\left(\beta, \delta, \frac{\lambda}{v}\right), & \lambda>0, \\
T(\beta, \delta, \lambda) \leq T\left(\beta, \delta, \frac{\lambda}{v}\right), & \lambda>0,
\end{array}
$$

then $\beta=\delta$.

Proof. Easy to show on the lines of [24].

Now, we introduce the notion of Banach contraction in the sense of ONMSs.

Definition 10. Let $(E, R, S, T, *, \Delta, \perp)$ be an ONMS. A map $\psi: E \longrightarrow E$ is an orthogonal contraction if there exists $\theta \in(0,1)$, such that for every $\lambda>0$ and $\beta, \delta \in E$ with $\beta \perp \delta$, we have

$$
\begin{aligned}
& R(\psi \beta, \psi \delta, \theta \lambda) \geq R(\beta, \delta, \lambda), \\
& S(\psi \beta, \psi \delta, \theta \lambda) \leq S(\beta, \delta, \lambda), \\
& T(\psi \beta, \psi \delta, \theta \lambda) \leq T(\beta, \delta, \lambda) .
\end{aligned}
$$

Theorem 1. Let $(E, R, S, T, *, \Delta, \perp)$ be an O-complete NMS, such that

$$
\begin{array}{r}
\lim _{\lambda \longrightarrow \infty} R(\beta, \delta, \lambda)=1, \\
\lim _{\lambda \longrightarrow \infty} S(\beta, \delta, \lambda)=0, \\
\lim _{\lambda \longrightarrow \infty} T(\beta, \delta, \lambda)=0, \\
\forall \beta, \delta \in E .
\end{array}
$$

Let $\psi: E \longrightarrow E$ be an $\perp$-continuous, $\perp$-contraction, and $\perp$-preserving mapping. Thus, $\psi$ has a unique FP, say $\beta_{*} \in E$. Furthermore,

$$
\begin{aligned}
\lim _{n \longrightarrow \infty} R\left(\psi^{n} \beta, \beta_{*}, \lambda\right) & =1, \\
\lim _{n \longrightarrow \infty} S\left(\psi^{n} \beta, \beta_{*}, \lambda\right) & =0, \\
\lim _{n \longrightarrow \infty} T\left(\psi^{n} \beta, \beta_{*}, \lambda\right) & =0, \\
\text { for all } \beta & \in E \text { and } \lambda>0 .
\end{aligned}
$$

Proof. Since $(E, R, S, T, *, \Delta, \perp)$ is an O-complete NMS, there exists $\beta_{0} \in E$, such that

$$
\beta_{0} \perp \delta, \quad \text { for all } \delta \in E .
$$

That is, $\beta_{0} \perp \psi \beta_{0}$. Take

$$
\beta_{n}=\psi^{n} \beta_{0}=\psi \beta_{n-1}, \quad \text { for all } n \in S .
$$

Since $\psi$ is $\perp$-preserving, $\left\{\beta_{n}\right\}$ is an O-sequence. Now, since $\psi$ is an $\perp$-contraction, we can get

$$
R\left(\beta_{n+1}, \beta_{n}, \theta \lambda\right)=R\left(\psi \beta_{n}, \psi \beta_{n-1}, \theta \lambda\right) \geq R\left(\beta_{n}, \beta_{n-1}, \lambda\right),
$$

for all $n \in S$ and $\lambda>0$. Note that $R$ is nondecreasing on $(0, \infty)$. Therefore, by applying the above expression, we can deduce

$$
\begin{aligned}
R\left(\beta_{n+1}, \beta_{n}, \lambda\right) & \geq R\left(\beta_{n+1}, \beta_{n}, \theta \lambda\right)=R\left(\psi \beta_{n}, \psi \beta_{n-1}, \theta \lambda\right) \geq R\left(\beta_{n}, \beta_{n-1}, \lambda\right) \\
& =R\left(\psi \beta_{n-1}, \psi \beta_{n-2}, \lambda\right) \geq R\left(\beta_{n-1}, \beta_{n-2}, \frac{\lambda}{\theta}\right) \geq \cdots \geq R\left(\beta_{1}, \beta_{0}, \frac{\lambda}{\theta^{n}}\right),
\end{aligned}
$$

for all $n \in S$ and $\lambda>0$. Thus, from (13) and (B4), we have

$$
\begin{aligned}
R\left(\beta_{n}, \beta_{n+\alpha}, \lambda\right) & \geq R\left(\beta_{n}, \beta_{n+1}, \frac{\lambda}{2}\right) * R\left(\beta_{n+1}, \beta_{n+\alpha}, \frac{\lambda}{2}\right) \\
& \geq R\left(\beta_{n}, \beta_{n+1}, \frac{\lambda}{2}\right) * R\left(\beta_{n+1}, \beta_{n+2}, \frac{\lambda}{2^{2}}\right) * R\left(\beta_{n+2}, \beta_{n+3}, \frac{\lambda}{2^{3}}\right) * \cdots * R\left(\beta_{n+\alpha-1}, \beta_{n+\alpha}, \frac{\lambda}{2^{n+\alpha}}\right) \\
& \geq R\left(\beta_{1}, \beta_{0}, \frac{\lambda}{2 \theta^{n}}\right) * R\left(\beta_{1}, \beta_{0}, \frac{\lambda}{2^{2} \theta^{n}}\right) * \cdots * R\left(\beta_{1}, \beta_{0}, \frac{\lambda}{2^{n+\alpha} \theta^{n}}\right) .
\end{aligned}
$$


We know that $\lim _{\lambda \longrightarrow \infty} R(\beta, \delta, \lambda)=1$, for all $\beta, \delta \in E$ and $\lambda>0$. So, from (17), we get

$$
\begin{aligned}
& \lim _{n \longrightarrow \infty} R\left(\beta_{n}, \beta_{n+\alpha}, \lambda\right) \geq 1 * 1 * \ldots * 1=1, \\
& S\left(\beta_{n+1}, \beta_{n}, \theta \lambda\right)=S\left(\psi \beta_{n}, \psi \beta_{n-1}, \theta \lambda\right) \leq S\left(\beta_{n}, \beta_{n-1}, \lambda\right),
\end{aligned}
$$

for all $n \in S$ and $\lambda>0$. Therefore, by applying the above expression, we can deduce

$$
\begin{aligned}
S\left(\beta_{n+1}, \beta_{n}, \lambda\right) & \leq S\left(\beta_{n+1}, \beta_{n}, \theta \lambda\right)=S\left(\psi \beta_{n}, \psi \beta_{n-1}, \theta \lambda\right) \leq S\left(\beta_{n}, \beta_{n-1}, \lambda\right) \\
& =S\left(\psi \beta_{n-1}, \psi \beta_{n-2}, \lambda\right) \leq S\left(\beta_{n-1}, \beta_{n-2}, \frac{\lambda}{\theta}\right) \leq \cdots \leq S\left(\beta_{1}, \beta_{0}, \frac{\lambda}{\theta^{n}}\right),
\end{aligned}
$$

for all $n \in S$ and $\lambda>0$. Thus, from (19) and (B10), we have

$$
\begin{aligned}
S\left(\beta_{n}, \beta_{n+\alpha}, \lambda\right) & \leq S\left(\beta_{n}, \beta_{n+1}, \frac{\lambda}{2}\right) \Delta S\left(\beta_{n+1}, \beta_{n+\alpha}, \frac{\lambda}{2}\right) \\
& \leq S\left(\beta_{n}, \beta_{n+1}, \frac{\lambda}{2}\right) \Delta S\left(\beta_{n+1}, \beta_{n+2}, \frac{\lambda}{2^{2}}\right) \Delta S\left(\beta_{n+2}, \beta_{n+3}, \frac{\lambda}{2^{3}}\right) \Delta \cdots \Delta S\left(\beta_{n+\alpha-1}, \beta_{n+\alpha}, \frac{\lambda}{2^{n+\alpha}}\right) \\
& \leq S\left(\beta_{1}, \beta_{0}, \frac{\lambda}{2 \theta^{n}}\right) \Delta S\left(\beta_{1}, \beta_{0}, \frac{\lambda}{2^{2} \theta^{n}}\right) \Delta \cdots \Delta S\left(\beta_{1}, \beta_{0}, \frac{\lambda}{2^{n+\alpha} \theta^{n}}\right) .
\end{aligned}
$$

We know that $\lim _{\lambda \longrightarrow \infty} S(\beta, \delta, \lambda)=0$ for all $\beta, \delta \in E$ and $\lambda>0$. So, from (20), we get

$$
\begin{aligned}
\lim _{n \longrightarrow \infty} S\left(\beta_{n}, \beta_{n+\alpha}, \lambda\right) \leq 0 \Delta 0 \Delta \cdots \Delta 0=0, & \\
T\left(\beta_{n+1}, \beta_{n}, \theta \lambda\right) & =T\left(\psi \beta_{n}, \psi \beta_{n-1}, \theta \lambda\right) \leq T\left(\beta_{n}, \beta_{n-1}, \lambda\right),
\end{aligned}
$$

for all $n \in S$ and $\lambda>0$. Therefore, by applying the above expression, we can deduce

$$
\begin{aligned}
T\left(\beta_{n+1}, \beta_{n}, \lambda\right) & \leq T\left(\beta_{n+1}, \beta_{n}, \theta \lambda\right)=T\left(\psi \beta_{n}, \psi \beta_{n-1}, \theta \lambda\right) \leq T\left(\beta_{n}, \beta_{n-1}, \lambda\right) \\
& =T\left(\psi \beta_{n-1}, \psi \beta_{n-2}, \lambda\right) \leq T\left(\beta_{n-1}, \beta_{n-2}, \frac{\lambda}{\theta}\right) \leq \cdots \leq T\left(\beta_{1}, \beta_{0}, \frac{\lambda}{\theta^{n}}\right),
\end{aligned}
$$

for all $n \in S$ and $\lambda>0$. Thus, from (22) and (B15), we have

$$
\begin{aligned}
T\left(\beta_{n}, \beta_{n+\alpha}, \lambda\right) & \leq T\left(\beta_{n}, \beta_{n+1}, \frac{\lambda}{2}\right) \Delta T\left(\beta_{n+1}, \beta_{n+\alpha}, \frac{\lambda}{2}\right) \\
& \leq T\left(\beta_{n}, \beta_{n+1}, \frac{\lambda}{2}\right) \Delta T\left(\beta_{n+1}, \beta_{n+2}, \frac{\lambda}{2^{2}}\right) \Delta T\left(\beta_{n+2}, \beta_{n+3}, \frac{\lambda}{2^{3}}\right) \Delta \cdots \Delta T\left(\beta_{n+\alpha-1}, \beta_{n+\alpha}, \frac{\lambda}{2^{n+\alpha}}\right) \\
& \leq T\left(\beta_{1}, \beta_{0}, \frac{\lambda}{2 \theta^{n}}\right) \Delta T\left(\beta_{1}, \beta_{0}, \frac{\lambda}{2^{2} \theta^{n}}\right) \Delta \cdots \Delta T\left(\beta_{1}, \beta_{0}, \frac{\lambda}{2^{n+\alpha} \theta^{n}}\right) .
\end{aligned}
$$


We know that $\lim _{\lambda \rightarrow \infty} T(\beta, \delta, \lambda)=0$, for all $\beta, \delta \in E$, and $\lambda>0$. So, from (23), we get

$$
\lim _{n \longrightarrow \infty} T\left(\beta_{n}, \beta_{n+\alpha}, \lambda\right) \leq 0 \Delta 0 \Delta \cdots \Delta 0=0 .
$$

So, $\left\{\beta_{n}\right\}$ is a Cauchy $\mathrm{O}$-sequence. The $\mathrm{O}$-completeness of the NMS $(E, R, S, T, *, \Delta, \perp)$ ensures that there exists $\beta_{*} \in E$, such that $R\left(\beta_{n}, \beta_{*}, \lambda\right) \longrightarrow 1, S\left(\beta_{n}, \beta_{*}, \lambda\right) \longrightarrow 0$, and $T\left(\beta_{n}, \beta_{*}, \lambda\right) \longrightarrow 0$ as $n \longrightarrow+\infty$ for all $\lambda>0$. Now, since $\psi$ is an $\perp$-continuous mapping, $R\left(\beta_{n+1}, \psi \beta_{*}, \lambda\right)=$ $R\left(\psi \beta_{n}, \psi \beta_{*}, \lambda\right) \longrightarrow 1, \quad S\left(\beta_{n+1}, \psi \beta_{*}, \lambda\right)=S\left(\psi \beta_{n}, \psi \beta_{*}, \lambda\right)$ $\longrightarrow 0$, and $T\left(\beta_{n+1}, \psi \beta_{*}, \lambda\right)=T\left(\psi \beta_{n}, \psi \beta_{*}, \lambda\right) \longrightarrow 0$ as $n \longrightarrow+\infty$. Now, we have

$$
\begin{aligned}
& R\left(\beta_{*}, \psi \beta_{*}, \lambda\right) \geq R\left(\beta_{*}, \beta_{n+1}, \frac{\lambda}{2}\right) * R\left(\beta_{n+1}, \psi \beta_{*}, \frac{\lambda}{2}\right), \\
& S\left(\beta_{*}, \psi \beta_{*}, \lambda\right) \leq S\left(\beta_{*}, \beta_{n+1}, \frac{\lambda}{2}\right) \Delta S\left(\beta_{n+1}, \psi \beta_{*}, \frac{\lambda}{2}\right), \\
& T\left(\beta_{*}, \psi \beta_{*}, \lambda\right) \leq T\left(\beta_{*}, \beta_{n+1}, \frac{\lambda}{2}\right) \Delta T\left(\beta_{n+1}, \psi \beta_{*}, \frac{\lambda}{2}\right) .
\end{aligned}
$$

Taking limit as $n \longrightarrow+\infty$, we get $R\left(\beta_{*}, \psi \beta_{*}, \lambda\right)=1 * 1=1, \quad S\left(\beta_{*}, \psi \beta_{*}, \lambda\right)=0 \Delta 0=0, \quad$ and $T\left(\beta_{*}, \psi \beta_{*}, \lambda\right)=0 \Delta 0=0$, and hence, $\psi \beta_{*}=\beta_{*}$.

Now, we show the uniqueness of the FP of the mapping $\psi$. Assume that $\beta_{*}$ and $\delta_{*}$ are two FPs of $\psi$, such that $\beta_{*} \neq \delta_{*}$. We can get

$$
\begin{aligned}
& \beta_{0} \perp \beta_{*}, \\
& \beta_{0} \perp \delta_{*} .
\end{aligned}
$$

Since $T$ is $\perp$-preserving, one writes

$$
\begin{aligned}
& \psi^{n} \beta_{0} \perp \psi^{n} \beta_{*}, \\
& \psi^{n} \beta_{0} \perp \psi^{n} \delta_{*},
\end{aligned}
$$

for all $n \in S$. So from (8), we can derive

$$
\begin{aligned}
& R\left(\psi^{n} \beta_{0}, \psi^{n} \beta_{*}, \lambda\right) \geq R\left(\psi^{n} \beta_{0}, \psi^{n} \beta_{*}, \theta \lambda\right) \geq R\left(\beta_{0}, \beta_{*}, \frac{\lambda}{\theta^{n}}\right), \\
& R\left(\psi^{n} \beta_{0}, \psi^{n} \delta_{*}, \lambda\right) \geq R\left(\psi^{n} \beta_{0}, \psi^{n} \delta_{*}, \theta \lambda\right) \geq R\left(\beta_{0}, \delta_{*}, \frac{\lambda}{\theta^{n}}\right) .
\end{aligned}
$$

Therefore,

$$
\begin{aligned}
R\left(\beta_{*}, \delta_{*}, \lambda\right) & =R\left(\psi^{n} \beta_{*}, \psi^{n} \delta_{*}, \lambda\right) \\
& \geq R\left(\psi^{n} \beta_{0}, \psi^{n} \beta_{*}, \frac{\lambda}{2}\right) * R\left(\psi^{n} \beta_{0}, \psi^{n} \delta_{*}, \frac{\lambda}{2}\right) \\
& \geq R\left(\beta_{0}, \beta_{*}, \frac{\lambda}{2 \theta^{n}}\right) * R\left(\beta_{0}, \delta_{*}, \frac{\lambda}{2 \theta^{n}}\right) \longrightarrow 1, \quad \text { as } n \longrightarrow \infty
\end{aligned}
$$

So from (9), we can derive

$$
\begin{aligned}
& S\left(\psi^{n} \beta_{0}, \psi^{n} \beta_{*}, \lambda\right) \leq S\left(\psi^{n} \beta_{0}, \psi^{n} \beta_{*}, \theta \lambda\right) \leq S\left(\beta_{0}, \beta_{*}, \frac{\lambda}{\theta^{n}}\right), \\
& S\left(\psi^{n} \beta_{0}, \psi^{n} \delta_{*}, \lambda\right) \leq S\left(\psi^{n} \beta_{0}, \psi^{n} \delta_{*}, \theta \lambda\right) \leq S\left(\beta_{0}, \delta_{*}, \frac{\lambda}{\theta^{n}}\right) .
\end{aligned}
$$

Therefore,

$$
\begin{aligned}
S\left(\beta_{*}, \delta_{*}, \lambda\right) & =S\left(\psi^{n} \beta_{*}, \psi^{n} \delta_{*}, \lambda\right) \\
& \leq S\left(\psi^{n} \beta_{0}, \psi^{n} \beta_{*}, \frac{\lambda}{2}\right) \Delta S\left(\psi^{n} \beta_{0}, \psi^{n} \delta_{*}, \frac{\lambda}{2}\right) \\
& \leq S\left(\beta_{0}, \beta_{*}, \frac{\lambda}{2 \theta^{n}}\right) \Delta S\left(\beta_{0}, \delta_{*}, \frac{\lambda}{2 \theta^{n}}\right) \longrightarrow 0, \text { as } n \longrightarrow \infty .
\end{aligned}
$$

Similarly, from (10), we can derive

$$
\begin{aligned}
& T\left(\psi^{n} \beta_{0}, \psi^{n} \beta_{*}, \lambda\right) \leq T\left(\psi^{n} \beta_{0}, \psi^{n} \beta_{*}, \theta \lambda\right) \leq T\left(\beta_{0}, \beta_{*}, \frac{\lambda}{\theta^{n}}\right), \\
& T\left(\psi^{n} \beta_{0}, \psi^{n} \delta_{*}, \lambda\right) \leq T\left(\psi^{n} \beta_{0}, \psi^{n} \delta_{*}, \theta \lambda\right) \leq T\left(\beta_{0}, \delta_{*}, \frac{\lambda}{\theta^{n}}\right) .
\end{aligned}
$$

Therefore,

$$
\begin{aligned}
T\left(\beta_{*}, \delta_{*}, \lambda\right) & =T\left(\psi^{n} \beta_{*}, \psi^{n} \delta_{*}, \lambda\right) \\
& \leq T\left(\psi^{n} \beta_{0}, \psi^{n} \beta_{*}, \frac{\lambda}{2}\right) \Delta T\left(\psi^{n} \beta_{0}, \psi^{n} \delta_{*}, \frac{\lambda}{2}\right) \\
& \leq T\left(\beta_{0}, \beta_{*}, \frac{\lambda}{2 \theta^{n}}\right) \Delta T\left(\beta_{0}, \delta_{*}, \frac{\lambda}{2 \theta^{n}}\right) \longrightarrow 0, \quad \text { as } n \longrightarrow \infty
\end{aligned}
$$

So, $\beta_{*}=\delta_{*}$; hence, $\beta_{*}$ is the unique FP.

Corollary 1. Let $(E, R, S, T, *, \Delta, \perp)$ be an O-complete NMS. Let $\psi: E \longrightarrow E$ be $\perp$-contraction and $\perp$-preserving. Suppose that if $\left\{\beta_{n}\right\}$ is an O-sequence with $\beta_{n} \longrightarrow \beta \in E$, then $\beta \perp \beta_{n}$ for all $n \in \mathbb{N}$. Then, $\psi$ has a unique $F P$, say $\beta_{*} \in E$. Furthermore, $\quad \lim _{n \rightarrow \infty} R\left(\psi^{n} \beta, \beta_{*}, \lambda\right)=1, \quad \lim _{n \longrightarrow \infty} S\left(\psi^{n}\right.$ $\left.\beta, \beta_{*}, \lambda\right)=0$, and $\lim _{n \longrightarrow \infty} T\left(\psi^{n} \beta, \beta_{*}, \lambda\right)=0$, for all $\beta \in E$ and $\lambda>0$.

Proof. See Appendix.

Example 4. Let $E=[-2,2]$. We define a binary relation $\perp$ by

$$
\beta \perp \delta \Longleftrightarrow \beta+\delta \geq 0
$$

Define an ONMS as in Example 2 by 


$$
\begin{aligned}
& R(\beta, \delta, \lambda)= \begin{cases}1, & \text { if } \beta=\delta, \\
\frac{\lambda}{\lambda+\max \{\beta, \delta\}}, & \text { if otherwise, }\end{cases} \\
& S(\beta, \delta, \lambda)= \begin{cases}0, & \text { if } \beta=\delta, \\
\frac{\max \{\beta, \delta\}}{\lambda+\max \{\beta, \delta\}}, & \text { if otherwise, }\end{cases} \\
& T(\beta, \delta, \lambda)= \begin{cases}0, & \text { if } \beta=\delta, \\
\frac{\max \{\beta, \delta\}}{\lambda}, & \text { if otherwise, }\end{cases}
\end{aligned}
$$

for all $\beta, \delta \in E, \lambda>0$, with the CTN $a * b=a \Delta b$ and CTCN $a \Delta b=\max \{a, b\}$. Then, $(E, R, S, T, *, \Delta, \perp)$ is an O-complete NMS. Define $\psi: E \longrightarrow E$ by

$$
\psi \beta= \begin{cases}\frac{\beta}{4}, & \beta \in[-2,0], \\ 0, & \beta \in(0,2] .\end{cases}
$$

Then, the following cases are satisfied.

(1) If $\beta \in[-2,0]$ and $\delta \in(0,2]$, then $\psi \beta=(\beta / 4)$ and $\psi \delta=0$

(2) If $\beta, \delta \in[-2,0]$, then $\psi \beta=(\beta / 4)$ and $\psi \delta=(\delta / 4)$

(3) If $\beta, \delta \in(0,2]$, then $\psi \beta=0$ and $\psi \delta=0$

(4) If $\beta \in(0,2]$ and $\delta \in[-2,0]$, then $\psi \beta=0$ and $\psi \delta=(\delta / 4)$

This clearly implies that $\psi \beta+\psi \delta \geq 0$. Hence, $\psi$ is $\perp--$ preserving. We can easily see that if $\lim _{n \longrightarrow \infty} R\left(\beta_{n}, \beta, \lambda\right)=1$, then $\lim _{n \longrightarrow \infty} R\left(\psi \beta_{n}, \psi \beta, \lambda\right)=1, \lim _{n \rightarrow \infty} S\left(\beta_{n}, \beta, \lambda\right)=0$, then $\lim _{n \rightarrow \infty} S\left(\psi \beta_{n}, \psi \beta, \lambda\right)=0$ and $\lim _{n \rightarrow \infty} T\left(\beta_{n}, \beta, \lambda\right)=0$, then $\lim _{n \longrightarrow \infty} T\left(\psi \beta_{n}, \psi \beta, \lambda\right)=0$ for all $\beta \in E$ and $\lambda>0$. Hence, $\psi$ is $\perp$-continuous.

The above cases $1-4$ for $\theta \in[(1 / 2), 1)$ are satisfied contractive conditions:

$$
\begin{gathered}
R(\psi \beta, \psi \delta, \theta \lambda) \geq R(\beta, \delta, \lambda), \\
S(\psi \beta, \psi \delta, \theta \lambda) \leq S(\beta, \delta, \lambda), \\
T(\psi \beta, \psi \delta, \theta \lambda) \leq T(\beta, \delta, \lambda) .
\end{gathered}
$$

All conditions of Theorem 1 are satisfied. Also, 0 is the unique FP of $\psi$.

Theorem 2. Let $(E, R, S, T, *, \Delta, \perp)$ be an O-complete NMS, such that

$$
\begin{aligned}
\lim _{\lambda \longrightarrow \infty} R(\beta, \delta, \lambda) & =1, \\
\lim _{\lambda \longrightarrow \infty} S(\beta, \delta, \lambda) & =0, \\
\forall \beta, \delta & \in E \text { and } \lambda>0 .
\end{aligned}
$$

Let $\psi: E \longrightarrow E$ be $\perp$-continuous, $\perp$-contraction, and $\perp$-preserving. Assume that there exist $\theta \in(0,1)$ and $\lambda>0$, such that

$$
\begin{gathered}
R(\psi \beta, \psi \delta, \theta \lambda) \geq \min \{R(\psi \beta, \beta, \lambda), R(\psi \delta, \delta, \lambda)\}, \\
S(\psi \beta, \psi \delta, \theta \lambda) \leq \min \{S(\psi \beta, \beta, \lambda), S(\psi \delta, \delta, \lambda)\}, \\
T(\psi \beta, \psi \delta, \theta \lambda) \leq \min \{T(\psi \beta, \beta, \lambda), T(\psi \delta, \delta, \lambda)\},
\end{gathered}
$$

for all $\beta, \delta \in E, \lambda>0$. Then, $\psi$ has a unique $F P$, so $\beta_{*} \in E$. Furthermore, $\quad \lim _{n \longrightarrow \infty} R\left(\psi^{n} \beta, \beta_{*}, \lambda\right)=1, \quad \lim _{n \rightarrow \infty} S$ $\left(\psi^{n} \beta, \beta_{*}, \lambda\right)=0$, and $\lim _{n \longrightarrow \infty} T\left(\psi^{n} \beta, \beta_{*}, \lambda\right)=0$ for all $\beta \in E$ and $\lambda>0$.

Proof. See Appendix.

Corollary 2. Let $(E, R, S, *, \Delta, \perp)$ be an O-complete IFMS and $\psi: E \longrightarrow E$ be an $\perp$-continuous and $\perp$-preserving mapping. Assume that there exists $\theta \in(0,1)$, so that for all $\lambda>0$,

$R(\psi \beta, \psi \delta, \theta \lambda) \geq \min \{R(\psi \beta, \beta, \lambda), R(\psi \delta, \delta, \lambda), R(\beta, \delta, \lambda)\}$, $S(\psi \beta, \psi \delta, \theta \lambda) \leq \min \{S(\psi \beta, \beta, \lambda), S(\psi \delta, \delta, \lambda), S(\beta, \delta, \lambda)\}$,

$T(\psi \beta, \psi \delta, \theta \lambda) \leq \min \{T(\psi \beta, \beta, \lambda), T(\psi \delta, \delta, \lambda), T(\beta, \delta, \lambda)\}$.

Then, $\psi$ has a unique FP.

Proof. It follows from Theorems 1 and 2 .

Example 5. Let $E=[-2,2]$ and define a binary relation $\perp$ by

$$
\beta \perp \delta \Longleftrightarrow \beta+\delta \geq 0 \text {. }
$$

Define $R$ and $S$ by

$$
\begin{aligned}
& R(\beta, \delta, \lambda)= \begin{cases}1, & \text { if } \beta=\delta, \\
\frac{\lambda}{\lambda+\max \{\beta, \delta\}}, & \text { otherwise, }\end{cases} \\
& S(\beta, \delta, \lambda)= \begin{cases}0, & \text { if } \beta=\delta, \\
\frac{\max \{\beta, \delta\}}{\lambda+\max \{\beta, \delta\}}, & \text { otherwise, }\end{cases}
\end{aligned}
$$

$$
T(\beta, \delta, \lambda)= \begin{cases}0, & \text { if } \beta=\delta, \\ \frac{\max \{\beta, \delta\}}{\lambda}, & \text { otherwise, }\end{cases}
$$

for all $\beta, \delta \in E$, and $\lambda>0$, with the CTN and CTCN, respectively: $a * b=a \cdot b, \quad a \Delta b=\max \{a, b\}, \quad$ then $(E, R, S, T, *, \Delta, \perp)$ is an O-complete NMS. Note that $\lim _{\lambda \longrightarrow \infty} R(\beta, \delta, \lambda)=1, \quad \lim _{\lambda \longrightarrow \infty} S(\beta, \delta, \lambda)=0, \quad$ and $\lim _{\lambda \longrightarrow \infty} T(\beta, \delta, \lambda)=0 \forall \beta, \delta \in E$. Define $\psi: E \longrightarrow E$ by 


$$
\psi \beta= \begin{cases}\frac{\beta}{4}, & \beta \in\left[-2, \frac{2}{3}\right], \\ 1-\beta, & \beta \in\left(\frac{2}{3}, 1\right], \\ \beta-\frac{1}{2}, & \beta \in(1,2] .\end{cases}
$$

We have the following cases:

(1) If $\beta, \delta \in[-2,(2 / 3)]$, then $\psi \beta=(\beta / 4)$ and $\psi \delta=(\delta / 4)$

(2) If $\beta, \delta \in((2 / 3), 1]$, then $\psi \beta=1-\beta$ and $\psi \delta=1-\delta$

(3) If $\beta, \delta \in(1,2]$, then $\psi \beta=\beta-(1 / 2) \quad$ and $\psi \delta=\delta-(1 / 2)$

(4) If $\beta \in[-2,(2 / 3)]$ and $\delta \in((2 / 3), 1]$, then $\psi \beta=(\beta / 4)$ and $\psi \delta=1-\delta$

(5) If $\beta \in[-2,(2 / 3)]$ and $\delta \in(1,2]$, then $\psi \beta=(\beta / 4)$ and $\psi \delta=\delta-(1 / 2)$

(6) If $\beta \in((2 / 3), 1]$ and $\delta \in(1,2]$, then $\psi \beta=1-\beta$ and $\psi \delta=\delta-(1 / 2)$

(7) If $\beta \in(1,2]$ and $\delta \in((2 / 3), 1]$, then $\psi \beta=\beta-(1 / 2)$ and $\psi \delta=1-\delta$

(8) If $\beta \in(1,2]$ and $\delta \in[-2,(2 / 3)]$, then $\psi \beta=\beta-(1 / 2)$ and $\psi \delta=(\delta / 4)$

(9) If $\beta \in((2 / 3), 1]$ and $\delta \in[-2,(2 / 3)]$, then $\psi \beta=1-\beta$ and $\psi \delta=(\delta / 4)$

Because $\beta \perp \delta \Longleftrightarrow \beta+\delta \geq 0$, it clearly implies that $\psi \beta+\psi \delta \geq 0$. Hence, $\psi$ is $\perp$-preserving. Let $\left\{\beta_{n}\right\}$ be an arbitrary $\mathrm{O}$-sequence in $E$ that converges to $\beta \in E$. We have

$$
\begin{aligned}
& \lim _{n \longrightarrow \infty} R\left(\beta_{n}, \beta, \lambda\right)=\lim _{n \longrightarrow \infty}\left\{\begin{array}{ll}
1, & \text { if } \beta=\delta, \\
\frac{\lambda}{\lambda+\max \left\{\beta_{n}, \beta\right\}}, & \text { otherwise }
\end{array}=1,\right. \\
& \lim _{n \rightarrow \infty} S\left(\beta_{n}, \beta, \lambda\right)=\lim _{n \longrightarrow \infty}\left\{\begin{array}{ll}
1, & \text { if } \beta=\delta, \\
\frac{\max \left\{\beta_{n}, \beta\right\}}{\lambda+\max \left\{\beta_{n}, \beta\right\}}, & \text { otherwise }
\end{array}=0,\right. \\
& \lim _{n \longrightarrow \infty} T\left(\beta_{n}, \beta, \lambda\right)=\lim _{n \longrightarrow \infty} \begin{cases}\frac{1,}{\max \left\{\beta_{n}, \beta\right\}} & \text { if } \beta=\delta, \\
\frac{\lambda}{1}, & \text { otherwise }\end{cases}
\end{aligned}
$$

Note that if $\lim _{n \rightarrow \infty} R\left(\beta_{n}, \beta, \lambda\right)=1, \quad \lim _{n \rightarrow \infty} S$ $\left(\beta_{n}, \beta, \lambda\right)=0$, and $\lim _{n \longrightarrow \infty} T\left(\beta_{n}, \beta, \lambda\right)=0$, then $\lim _{n \longrightarrow \infty}$ $R\left(\left(\psi \beta_{n}, \psi \beta, \lambda\right)=1, \lim _{n \rightarrow \infty} S\left(\psi \beta_{n}, \psi \beta, \lambda\right)=0\right.$, and $\lim _{n \rightarrow \infty}$ $T\left(\psi \beta_{n}, \psi \beta, \lambda\right)=0$ for all $\beta \in E$ and $\lambda>0$. Hence, $\psi$ is orthogonal continuous. The case $\beta=\delta$ is clear. Let $\beta \neq \delta$. We have
$R(\psi \beta, \psi \delta, \theta \lambda) \geq \min \{R(\psi \beta, \beta, \lambda), R(\psi \delta, \delta, \lambda)\}$,

$S(\psi \beta, \psi \delta, \theta \lambda) \leq \min \{S(\psi \beta, \beta, \lambda), S(\psi \delta, \delta, \lambda)\}$,

$T(\psi \beta, \psi \delta, \theta \lambda) \leq \min \{T(\psi \beta, \beta, \lambda), T(\psi \delta, \delta, \lambda)\}$.

Indeed, it is satisfied for all above 9 cases. But, $\psi$ is not a contraction. Assume

$$
\begin{aligned}
\min \{R(\psi \beta, \beta, \lambda), R(\psi \delta, \delta, \lambda)\} & =R(\psi \beta, \beta, \lambda), \\
\min \{S(\psi \beta, \beta, \lambda), S(\psi \delta, \delta, \lambda)\} & =S(\psi \beta, \beta, \lambda), \\
\min \{T(\psi \beta, \beta, \lambda), T(\psi \delta, \delta, \lambda)\} & =T(\psi \beta, \beta, \lambda),
\end{aligned}
$$

then for $\beta=-1, \delta=-2$, we have

$$
\begin{aligned}
& R(\psi \beta, \psi \delta, \theta \lambda)=\frac{\theta \lambda}{\theta \lambda+\max \{(\beta / 4),(\delta / 4)\}}=\frac{4 \theta \lambda}{4 \theta \lambda-1} \geq 1, \\
& S(\psi \beta, \psi \delta, \theta \lambda)=\frac{\max \{(\beta / 4),(\delta / 4)\}}{\theta \lambda+\max \{(\beta / 4),(\delta / 4)\}}=\frac{-1}{4 \theta \lambda-1} \leq 0, \\
& S(\psi \beta, \psi \delta, \theta \lambda)=\frac{\max \{(\beta / 4),(\delta / 4)\}}{\theta \lambda}=\frac{-1}{4 \theta \lambda} \leq 0 .
\end{aligned}
$$

It is a contradiction. Hence, all the conditions of Theorem 2 are satisfied and 0 is the unique FP of $\psi$.

Definition 11. Let $(E, R, S, T, *, \Delta, \perp)$ be an ONMS. A mapping $\psi: E \longrightarrow E$ is named to be an NS $\perp$-contractive if $\exists \theta \in(0,1)$, so that

$$
\begin{gathered}
\frac{1}{R(\psi \beta, \psi \delta, \lambda)}-1 \leq \theta\left[\frac{1}{R(\beta, \delta, \lambda)}-1\right], \\
S(\psi \beta, \psi \delta, \lambda) \leq \theta S(\beta, \delta, \lambda), \\
T(\psi \beta, \psi \delta, \lambda) \leq \theta T(\beta, \delta, \lambda),
\end{gathered}
$$

for all $\beta, \delta \in E$ and $\lambda>0$. Here, $\theta$ is called the NS $\perp$-contractive constant of $\psi$.

Theorem 3. Let $(E, R, S, T, *, \Delta, \perp)$ be an O-complete NMS, such that

$$
\begin{array}{r}
\lim _{\lambda \longrightarrow \infty} R(\beta, \delta, \lambda)=1, \\
\lim _{\lambda \longrightarrow \infty} S(\beta, \delta, \lambda)=0, \\
\lim _{\lambda \longrightarrow \infty} T(\beta, \delta, \lambda)=0, \\
\forall \beta, \delta \in E .
\end{array}
$$

Let $\psi: E \longrightarrow E$ be an $\perp$-continuous, NS $\perp$-contraction, and $\perp$-preserving mapping. Thus, $\psi$ has a FP, say $v \in E$, $R(v, v, \lambda)=1, S(v, v, \lambda)=0$, and $T(v, v, \lambda)=0$ for all $\lambda>0$.

Proof. Let $(E, R, S, T, *, \Delta, \perp)$ be an O-complete NMS. For an arbitrary $\beta_{0} \in E$, 


$$
\begin{aligned}
& \beta_{0} \perp \delta, \\
& \forall \delta \in E .
\end{aligned}
$$

That is, $\beta_{0} \perp \psi \beta_{0}$. Consider

$$
\beta_{n}=\psi^{n} \beta_{0}=\psi \beta_{n-1}, \quad \text { for all } n \in \mathbb{N} \text {. }
$$

$$
\begin{aligned}
\frac{1}{R\left(\beta_{n}, \beta_{n+1}, \lambda\right)}-1 & =\frac{1}{R\left(\psi \beta_{n-1}, \psi \beta_{n}, \lambda\right)}-1 \leq \theta\left[\frac{1}{R\left(\beta_{n-1}, \beta_{n}, \lambda\right)}-1\right], \\
S\left(\beta_{n}, \beta_{n+1}, \lambda\right) & =S\left(\psi \beta_{n-1}, \psi \beta_{n}, \lambda\right) \leq \theta S\left(\beta_{n-1}, \beta_{n}, \lambda\right) \\
T\left(\beta_{n}, \beta_{n+1}, \lambda\right) & =T\left(\psi \beta_{n-1}, \psi \beta_{n}, \lambda\right) \leq \theta T\left(\beta_{n-1}, \beta_{n}, \lambda\right) .
\end{aligned}
$$

We have

$$
\begin{aligned}
\frac{1}{R\left(\beta_{n}, \beta_{n+1}, \lambda\right)} & \leq \frac{\theta}{R\left(\beta_{n-1}, \beta_{n}, \lambda\right)}+(1-\theta), \quad \forall \lambda>0 \\
& =\frac{\theta}{R\left(\psi \beta_{n-2}, \psi \beta_{n-1}, \lambda\right)}+(1-\theta) \leq \frac{\theta^{2}}{R\left(\beta_{n-2}, \beta_{n-1}, \lambda\right)}+\theta(1-\theta)+(1-\theta) .
\end{aligned}
$$

Continuing in this way, we get

$$
\begin{aligned}
\frac{1}{R\left(\beta_{n}, \beta_{n+1}, \lambda\right)} & \leq \frac{\theta^{n}}{R\left(\beta_{0}, \beta_{1}, \lambda\right)}+\theta^{n-1}(1-\theta)+\theta^{n-2}(1-\theta)+\cdots+\theta(1-\theta)+(1-\theta) \\
& \leq \frac{\theta^{n}}{R\left(\beta_{0}, \beta_{1}, \lambda\right)}+\left(\theta^{n-1}+\theta^{n-2}+\cdots+1\right)(1-\theta) \\
& \leq \frac{\theta^{n}}{R\left(\beta_{0}, \beta_{1}, \lambda\right)}+\left(1-\theta^{n}\right) .
\end{aligned}
$$

We have

$$
\begin{aligned}
\frac{1}{\left(\theta^{n} / R\left(\beta_{0}, \beta_{1}, \lambda\right)\right)+\left(1-\theta^{n}\right)} & \leq R\left(\beta_{n}, \beta_{n+1}, \lambda\right), \quad \forall \lambda>0, n \in \mathbb{N}, \\
S\left(\beta_{n}, \beta_{n+1}, \lambda\right) & =S\left(\psi \beta_{n-1}, \psi \beta_{n}, \lambda\right) \leq \theta S\left(\beta_{n-1}, \beta_{n}, \lambda\right)=\theta S\left(\psi \beta_{n-2}, \psi \beta_{n-1}, \lambda\right) \\
& \leq \theta^{2} S\left(\beta_{n-2}, \beta_{n-1}, \lambda\right) \leq \cdots \leq \theta^{n} S\left(\beta_{0}, \beta_{1}, \lambda\right), \quad \forall \lambda>0, n \in \mathbb{N}, \\
T\left(\beta_{n}, \beta_{n+1}, \lambda\right) & =T\left(\psi \beta_{n-1}, \psi \beta_{n}, \lambda\right) \leq \theta T\left(\beta_{n-1}, \beta_{n}, \lambda\right)=\theta T\left(\psi \beta_{n-2}, \psi \beta_{n-1}, \lambda\right) \\
& \leq \theta^{2} T\left(\beta_{n-2}, \beta_{n-1}, \lambda\right) \leq \cdots \leq \theta^{n} T\left(\beta_{0}, \beta_{1}, \lambda\right), \quad \forall \lambda>0, n \in \mathbb{N} .
\end{aligned}
$$

Now, for $m \geq 1$ and $n \in \mathbb{N}$, we have 


$$
\begin{aligned}
R\left(\beta_{n}, \beta_{n+m}, \lambda\right) \geq & R\left(\beta_{n}, \beta_{n+1}, \frac{\lambda}{2}\right) * R\left(\beta_{n+1}, \beta_{n+m}, \frac{\lambda}{2}\right) \\
\geq & R\left(\beta_{n}, \beta_{n+1}, \frac{\lambda}{2}\right) * R\left(\beta_{n+1}, \beta_{n+2}, \frac{\lambda}{2^{2}}\right) \\
& * R\left(\beta_{n+2}, \beta_{n+m}, \frac{\lambda}{2^{2}}\right) .
\end{aligned}
$$

$$
\begin{aligned}
R\left(\beta_{n}, \beta_{n+m}, \lambda\right) & \geq R\left(\beta_{n}, \beta_{n+1}, \frac{\lambda}{2}\right) * R\left(\beta_{n+1}, \beta_{n+2}, \frac{\lambda}{2^{2}}\right) * \cdots * R\left(\beta_{n+m-1}, \beta_{n+m}, \frac{\lambda}{2^{m-1}}\right) \\
S\left(\beta_{n}, \beta_{n+p}, \lambda\right) & \leq S\left(\beta_{n}, \beta_{n+1}, \frac{\lambda}{2}\right) \Delta S\left(\beta_{n+1}, \beta_{n+p}, \frac{\lambda}{2}\right) \\
& \leq S\left(\beta_{n}, \beta_{n+1}, \frac{\lambda}{2}\right) \Delta S\left(\beta_{n+1}, \beta_{n+2}, \frac{\lambda}{2^{2}}\right) \Delta S\left(\beta_{n+2}, \beta_{n+p}, \frac{\lambda}{2^{2}}\right) .
\end{aligned}
$$

Continuing in this way, we get

$$
\begin{aligned}
S\left(\beta_{n}, \beta_{n+p}, \lambda\right) & \leq S\left(\beta_{n}, \beta_{n+1}, \frac{\lambda}{2}\right) \Delta S\left(\beta_{n+1}, \beta_{n+2}, \frac{\lambda}{2^{2}}\right) \Delta \cdots \Delta S\left(\beta_{n+p-1}, \beta_{n+p}, \frac{\lambda}{2^{p-1}}\right) \\
T\left(\beta_{n}, \beta_{n+p}, \lambda\right) & \leq T\left(\beta_{n}, \beta_{n+1}, \frac{\lambda}{2}\right) \Delta T\left(\beta_{n+1}, \beta_{n+p}, \frac{\lambda}{2}\right) \\
& \leq T\left(\beta_{n}, \beta_{n+1}, \frac{\lambda}{2}\right) \Delta T\left(\beta_{n+1}, \beta_{n+2}, \frac{\lambda}{2^{2}}\right) \Delta T\left(\beta_{n+2}, \beta_{n+p}, \frac{\lambda}{2^{2}}\right) .
\end{aligned}
$$

Continuing in this way, we get

$$
T\left(\beta_{n}, \beta_{n+p}, \lambda\right) \leq T\left(\beta_{n}, \beta_{n+1}, \frac{\lambda}{2}\right) \Delta T\left(\beta_{n+1}, \beta_{n+2}, \frac{\lambda}{2^{2}}\right) \Delta \cdots \Delta T\left(\beta_{n+p-1}, \beta_{n+p}, \frac{\lambda}{2^{p-1}}\right)
$$

By using (55) in the above inequality, we have

$$
\begin{aligned}
R\left(\beta_{n}, \beta_{n+m}, \lambda\right) \geq & \frac{1}{\left(\theta^{n} / R\left(\beta_{0}, \beta_{1},(\lambda / 2)\right)\right)+\left(1-\theta^{n}\right)} * \frac{1}{\left(\theta^{n+1} / R\left(\beta_{0}, \beta_{1},\left(\lambda / 2^{2}\right)\right)\right)+\left(1-\theta^{n+1}\right)} \\
& * \cdots * \frac{1}{\left(\theta^{n+m-1} / R\left(\beta_{0}, \beta_{1},\left(\lambda / 2^{m-1}\right)\right)\right)+\left(1-\theta^{n+m-1}\right)} \\
\geq & \frac{1}{\left(\theta^{n} / R\left(\beta_{0}, \beta_{1},(\lambda / 2)\right)\right)+1} * \frac{1}{\left(\theta^{n+1} / R\left(\beta_{0}, \beta_{1},\left(\lambda / 2^{2}\right)\right)\right)+1} \\
& * \cdots * \frac{1}{\left(\theta^{n+m-1} / R\left(\beta_{0}, \beta_{1},\left(\lambda / 2^{m-1}\right)\right)\right)+1}
\end{aligned}
$$


using (56),

$$
S\left(\beta_{n}, \beta_{n+p}, \lambda\right) \leq S\left(\beta_{n}, \beta_{n+1}, \frac{\lambda}{2}\right) \Delta S\left(\beta_{n+1}, \beta_{n+2}, \frac{\lambda}{2^{2}}\right) \Delta \cdots \Delta S\left(\beta_{n+p-1}, \beta_{n+p}, \frac{\lambda}{2^{p-1}}\right),
$$

and using (57),

$$
T\left(\beta_{n}, \beta_{n+p}, \lambda\right) \leq T\left(\beta_{n}, \beta_{n+1}, \frac{\lambda}{2}\right) \Delta T\left(\beta_{n+1}, \beta_{n+2}, \frac{\lambda}{2^{2}}\right) \Delta \cdots \Delta T\left(\beta_{n+p-1}, \beta_{n+p}, \frac{\lambda}{2^{p-1}}\right),
$$

$\theta \in(0,1)$, we deduce from the above expression that

$$
\begin{aligned}
& \lim _{n \longrightarrow \infty} R\left(\beta_{n}, \beta_{n+m}, \lambda\right)=1, \\
& \lim _{n \longrightarrow \infty} S\left(\beta_{n}, \beta_{n+m}, \lambda\right)=0, \\
& \lim _{n \longrightarrow \infty} T\left(\beta_{n}, \beta_{n+m}, \lambda\right)=0, \\
& \text { for all } \lambda>0, m \geq 1 .
\end{aligned}
$$

Therefore, $\left\{\beta_{n}\right\}$ is a Cauchy O-sequence in $(E, R, S, T, *, \Delta, \perp)$. By the completeness of ( $E, R, S, T, *, \Delta, \perp)$, we know that $\psi$ is an $\perp$-continuous mapping and there exists $v \in E$, such that

$$
\begin{aligned}
& \lim _{n \longrightarrow \infty} R\left(\beta_{n+1}, v, \lambda\right)=\lim _{n \longrightarrow \infty} R\left(\psi \beta_{n}, \psi v, \lambda\right)=1, \quad \forall \lambda>0, \\
& \lim _{n \longrightarrow \infty} S\left(\beta_{n+1}, v, \lambda\right)=\lim _{n \longrightarrow \infty} S\left(\psi \beta_{n}, \psi v, \lambda\right)=0, \quad \forall \lambda>0,
\end{aligned}
$$

$$
\lim _{n \longrightarrow \infty} T\left(\beta_{n+1}, v, \lambda\right)=\lim _{n \longrightarrow \infty} T\left(\psi \beta_{n}, \psi v, \lambda\right)=0, \quad \forall \lambda>0 .
$$

Now, we prove that $v$ is a FP of $\psi$. For this, we obtain from (48) that

$$
\frac{1}{R\left(\psi \beta_{n}, \psi v, \lambda\right)}-1 \leq \theta\left[\frac{1}{R\left(\beta_{n}, v, \lambda\right)}-1\right]=\frac{\theta}{R\left(\beta_{n}, v, \lambda\right)}-\theta .
$$

That is,

$$
\frac{1}{\left(\theta / R\left(\beta_{n}, v, \lambda\right)\right)+1-\theta} \leq R\left(\psi \beta_{n}, \psi v, \lambda\right) .
$$

Using the above inequality, we obtain

$$
\begin{aligned}
R(v, \psi v, \lambda) & \geq R\left(v, \beta_{n+1}, \frac{\lambda}{2}\right) * R\left(\beta_{n+1}, \psi v, \frac{\lambda}{2}\right) \\
& =R\left(v, \beta_{n+1}, \frac{\lambda}{2}\right) * R\left(\psi \beta_{n}, \psi v, \frac{\lambda}{2}\right) \\
& \geq R\left(v, \beta_{n+1}, \frac{\lambda}{2}\right) * \frac{1}{\left(\theta / R\left(\beta_{n}, v, \lambda / 2\right)\right)+1-\theta},
\end{aligned}
$$

$S(w, v, \lambda)=S(\psi w, \psi v, \lambda) \leq \theta S(w, v, \lambda)<S(w, v, \lambda)$

$$
\begin{aligned}
& =S\left(w, \beta_{n+1}, \frac{\lambda}{2}\right) \Delta S\left(\psi \beta_{n}, \psi w, \frac{\lambda}{2}\right) \\
& \leq S\left(w, \beta_{n+1}, \frac{\lambda}{2}\right) \Delta \theta S\left(\beta_{n}, w, \frac{\lambda}{2}\right),
\end{aligned}
$$

$$
T(w, v, \lambda)=T(\psi w, \psi v, \lambda) \leq \theta T(w, v, \lambda)<T(w, v, \lambda)
$$

$$
\begin{aligned}
& =T\left(w, \beta_{n+1}, \frac{\lambda}{2}\right) \Delta T\left(\psi \beta_{n}, \psi w, \frac{\lambda}{2}\right) \\
& \leq T\left(w, \beta_{n+1}, \frac{\lambda}{2}\right) \Delta \theta T\left(\beta_{n}, w, \frac{\lambda}{2}\right) .
\end{aligned}
$$

Taking limit as $n \longrightarrow \infty$ and using (66)-(68) in the above expression, we get that $R(v, \psi v, \lambda)=1, S(v, \psi v, \lambda)=0$ and $T(v, \psi v, \lambda)=0$, that is, $\psi v=v$. Therefore, $v$ is a FP of $\psi$, $R(v, v, \lambda)=1, \quad S(v, v, \lambda)=0$, and $T(v, v, \lambda)=0$ for all $\lambda>0$.

Corollary 3. Let $(E, R, S, T, *, \Delta, \perp)$ be a O-complete NMS and $\psi: E \longrightarrow$ Esatisfy 


$$
\begin{gathered}
\frac{1}{R\left(\psi^{n} \beta, \psi^{n} \delta, \lambda\right)}-1 \leq \theta\left[\frac{1}{R(\beta, \delta, \lambda)}-1\right], \\
S\left(\psi^{n} \beta, \psi^{n} \delta, \lambda\right) \leq \theta S(\beta, \delta, \lambda), \\
T\left(\psi^{n} \beta, \psi^{n} \delta, \lambda\right) \leq \theta T(\beta, \delta, \lambda),
\end{gathered}
$$

for all $n \in \mathbb{N}, \beta, \delta \in E, \lambda>0$, where $0<\theta<1$. Then, $\psi$ has a $F P$.

Proof. $v \in E$ is the unique FP of $\psi^{n}$ by using Theorem 3 , and $R(v, v, \lambda)=1, S(v, v, \lambda)=0, T(v, v, \lambda), \forall \lambda>0, \psi v$ is also a FP of $\psi^{n}$ as $\psi^{n}(\psi v)=\psi v$. From Theorem 3, $\psi v=v$, where $v$ is a FP since the FP of $\psi$ is also a FP of $\psi^{n}$.

Example 6. Let $E=[-1,2]$ and define a binary relation $\perp$ by

$$
\beta \perp \delta \Longleftrightarrow \beta+\delta \geq 0 \text {. }
$$

Define $R, S, T$ by

$$
\begin{aligned}
& R(\beta, \delta, \lambda)= \begin{cases}1, & \text { if } \beta=\delta, \\
\frac{\lambda}{\lambda+\max \{\beta, \delta\}}, & \text { if otherwise, }\end{cases} \\
& S(\beta, \delta, \lambda)= \begin{cases}0, & \text { if } \beta=\delta, \\
1-\frac{\lambda}{\lambda+\max \{\beta, \delta\}}, & \text { if otherwise, }\end{cases} \\
& T(\beta, \delta, \lambda)= \begin{cases}0, & \text { if } \beta=\delta, \\
\frac{\max \{\beta, \delta\}}{\lambda}, & \text { if otherwise. }\end{cases}
\end{aligned}
$$

With CTN $a * b=a \cdot b$ and CTCN $a \Delta b=\max \{a, b\}$, $(E, R, S, T, *, \Delta, \perp)$ is an O-complete NMS. Also, observe that $\lim _{\lambda \longrightarrow \infty} R(\beta, \delta, \lambda)=1, \quad \lim _{\lambda \longrightarrow \infty} S(\beta, \delta, \lambda)=0$, and $\lim _{\lambda \rightarrow \infty} T(\beta, \delta, \lambda)=0, \forall \beta, \delta \in E$.

Define $\psi: E \longrightarrow E$ by

$$
\psi \beta= \begin{cases}2-\beta, & \beta \in[-1,1), \\ 1, & \beta \in[1,2] .\end{cases}
$$

Therefore, it will satisfy the following cases:

(1) If $\beta, \delta \in[-1,1)$, then $\psi \beta=2-\beta$ and $\psi \delta=2-\delta$

(2) If $\beta, \delta \in[1,2]$, then $\psi \beta=\psi \delta=1$

(3) If $\beta \in[-1,1)$ and $\delta \in[1,2]$, then $\psi \beta=2-\beta$ and $\psi \delta=1$

(4) If $\beta \in[1,2]$ and $\delta \in[-1,1)$, then $\psi \beta=1$ and $\psi \delta=2-\delta$

Because $\beta \perp \delta \Longleftrightarrow \beta+\delta \geq 0$, it clearly implies that $\psi \beta+\psi \delta \geq 0$. Hence, $\psi$ is $\perp$-preserving. Let $\left\{\beta_{n}\right\}$ be an arbitrary $\mathrm{O}$-sequence in $E$ that $\left\{\beta_{n}\right\}$ converges to $\beta \in E$.

$$
\begin{aligned}
& \lim _{n \longrightarrow \infty} R\left(\beta_{n}, \beta, \lambda\right)=1, \\
& \lim _{n \longrightarrow \infty} S\left(\beta_{n}, \beta, \lambda\right)=0, \\
& \lim _{n \longrightarrow \infty} T\left(\beta_{n}, \beta, \lambda\right)=0,
\end{aligned}
$$

as $\left\{\beta_{n}\right\}$ converges to $\beta$. We can easily see that if $\lim _{n \longrightarrow \infty} R\left(\beta_{n}, \beta, \lambda\right)=1, \lim _{n \longrightarrow \infty} S\left(\beta_{n}, \beta, \lambda\right)=0, \quad$ and $\lim _{n \rightarrow \infty} T\left(\beta_{n}, \beta, \lambda\right)=0$, then clearly $\lim _{n \rightarrow \infty} R\left(\psi \beta_{n}\right.$, $\psi \beta, \lambda)=1, \lim _{n \rightarrow \infty} S\left(\psi \beta_{n}, \psi \beta, \lambda\right)=0$, and $\lim _{n \longrightarrow \infty} T\left(\psi \beta_{n}\right.$, $\psi \beta, \lambda)=0$ for all $\beta \in E$ and $\lambda>0$. Hence, $\psi$ is orthogonal continuous. Also, above all cases satisfied NS $\perp$-contractive mapping

$$
\begin{gathered}
\frac{1}{R(\psi \beta, \psi \delta, \lambda)}-1 \leq \theta\left[\frac{1}{R(\beta, \delta, \lambda)}-1\right], \\
S(\psi \beta, \psi \delta, \lambda) \leq \theta S(\beta, \delta, \lambda), \\
T(\psi \beta, \psi \delta, \lambda) \leq \theta T(\beta, \delta, \lambda) .
\end{gathered}
$$

All conditions of Theorem 3 are satisfied and 1 is a FP of $\psi$.

\section{Conclusion}

Herein, we have introduced the notion of orthogonal neutrosophic metric space and investigated some new type fixed point theorems in this new setting. Moreover, we have provided nontrivial examples to demonstrate the viability of the proposed methods. Since our structure is more general than the class of neutrosophic metric spaces, our results and notions expand and generalize a number of previously published results. This study can be easily extended in the structure of orthogonal neutrosophic cone metric spaces, orthogonal neutrosophic triple partial metric spaces, orthogonal neutrosophic triple V-generalized metric spaces, orthogonal neutrosophic triple partial bipolar metric spaces, and orthogonal neutrosophic triple partial g-metric spaces.

\section{Appendix}

Definition A.1 (See [11]). A binary operation *: $[0,1] \times[0$, $1] \longrightarrow[0,1]$ is called a CTN if

$$
\pi^{*} \mu=\mu^{*} \pi, \quad(\forall) \pi, \mu \in[0,1] .
$$

$(1){ }^{*}$ is continuous;

$$
\begin{aligned}
\pi^{*} 1 & =\pi, \quad(\forall) \pi \in[0,1], \\
\left(\pi^{*} \mu\right)^{*} \rho & =\pi^{*}\left(\mu^{*} \rho\right), \quad(\forall) \pi, \mu, \rho \in[0,1] .
\end{aligned}
$$

(2) If $\pi \leq \rho$ and $\mu \leq \sigma$, with $\pi, \mu, \rho, \sigma \in[0,1]$, then $\pi^{*} \mu \leq \rho^{*} \sigma$.

Example A.1 (See [11]). Some fundamental examples of CTNs are $\pi^{*} \mu=\pi \cdot \mu, \pi^{*} \mu=\min \{\pi, \mu\} \quad$ and $\pi^{*} \mu=\max \{\pi+\mu-1,0\}$. 
Definition A.2 (See [11]). A binary operation $\bigcirc:[0,1] \times[0$, $1] \longrightarrow[0,1]$ is called a CTCN if it meets the following assertions:

$$
\pi \bigcirc \mu=\mu \bigcirc \pi, \quad \text { for all } \pi, \mu \in[0,1] .
$$

(a) $\bigcirc$ is continuous;

$$
\begin{gathered}
\pi \bigcirc 0 \\
(\pi \bigcirc \mu) \bigcirc \rho=\pi \bigcirc(\mu \bigcirc \rho), \quad \text { for all } \pi, \mu, \rho \in[0,1] .
\end{gathered}
$$

(b) If $\pi \leq \rho$ and $\mu \leq \sigma$, with $\pi, \mu, \rho, \sigma \in[0,1]$, then $\pi \bigcirc \mu \leq \rho \bigcirc \sigma$.

Example A.2 (See [11]). $\pi \bigcirc \mu=\max \{\pi, \mu\}$ and $\pi \bigcirc \mu=$ $\min \{\pi+\mu, 1\}$ are examples of CTCNs.

Definition A.3 (See [1]). A fuzzy set is a pair $(E, f)$, where $E$ is a nonempty set, $f: E \longrightarrow[0,1]$ is a membership function, and for each $\beta \in E, f(\beta)$ is called the grade of membership of $\beta$ in $(E, f)$.

Definition A.4 (See [18]). Let $E \neq \varnothing$ and $\partial \in E$. A neutrosophic set $G$ in $E$ is categorized by a truth-membership function, $R_{G}(\partial)$, an indeterminacy-membership function $S(\partial)$ and a falsity membership function $T_{G}(\partial)$. The functions $R_{G}(\partial), S_{G}(\partial)$, and $T_{G}(\partial)$ are real standard or nonstandard subsets of $] 0^{-}, 1^{+}\left[\right.$, that is, $\left.R_{G}(\partial): X \longrightarrow\right] 0^{-}, 1^{+}[$, $\left.S_{G}(\partial): X \longrightarrow\right] 0^{-}, 1^{+}\left[\right.$and $\left.T_{G}(\partial): X \longrightarrow\right] 0^{-}, 1^{+}[$. So,

$$
0^{-} \leq \sup R_{G}(\partial)+\sup S_{G}(\partial)+\sup T_{G}(\partial) \leq 3^{+} .
$$

Proof. of Corollary 1. We can similarly derive as in the proof of Theorem 1 that $\left\{\beta_{n}\right\}$ is a Cauchy $\mathrm{O}$-sequence, and so, it converges to $\beta_{*} \in E$. Hence, $\beta_{*} \perp \beta_{n}$ for all $n \in \mathbb{N}$. From (8), we can get

$$
\begin{aligned}
R\left(\psi \beta_{*}, \beta_{n+1}, \lambda\right) & =R\left(\psi \beta_{*}, \psi \beta_{n}, \lambda\right) \\
& \geq R\left(\psi \beta_{*}, \psi \beta_{n}, \lambda \theta\right) \geq R\left(\beta_{*}, \beta_{n}, \lambda\right) \\
\lim _{n \longrightarrow \infty} R\left(\psi \beta_{*}, \beta_{n+1}, \lambda\right) & =1 .
\end{aligned}
$$

Then, we can write

$$
R\left(\beta_{*}, \psi \beta_{*}, \lambda\right) \geq R\left(\beta_{*}, \beta_{n+1}, \frac{\lambda}{2}\right) * R\left(\beta_{n+1}, \psi \beta_{*}, \frac{\lambda}{2}\right)
$$

Taking limit as $n \longrightarrow+\infty$, we get $R\left(\beta_{*}, \psi \beta_{*}, \lambda\right)=1 * 1=1$, and from (9), we can get

$$
\begin{aligned}
S\left(\psi \beta_{*}, \beta_{n+1}, \lambda\right) & =S\left(\psi \beta_{*}, \psi \beta_{n}, \lambda\right) \leq S\left(\psi \beta_{*}, \psi \beta_{n}, \lambda \theta\right) \\
& \leq S\left(\beta_{*}, \beta_{n}, \lambda\right) \\
\lim _{n \longrightarrow \infty} S\left(\psi \beta_{*}, \beta_{n+1}, \lambda\right) & =0 .
\end{aligned}
$$

Then, we can write

$$
S\left(\beta_{*}, \psi \beta_{*}, \lambda\right) \leq S\left(\beta_{*}, \beta_{n+1}, \frac{\lambda}{2}\right) \Delta S\left(\beta_{n+1}, \psi \beta_{*}, \frac{\lambda}{2}\right) .
$$

Taking limit as $n \longrightarrow+\infty$, we get

$$
S\left(\beta_{*}, \psi \beta_{*}, \lambda\right)=0 \Delta 0=0
$$

and from (10), we can get

$$
\begin{aligned}
T\left(\psi \beta_{*}, \beta_{n+1}, \lambda\right) & =T\left(\psi \beta_{*}, \psi \beta_{n}, \lambda\right) \\
& \leq T\left(\psi \beta_{*}, \psi \beta_{n}, \lambda \theta\right) \leq T\left(\beta_{*}, \beta_{n}, \lambda\right) \\
\lim _{n \longrightarrow \infty} T\left(\psi \beta_{*}, \beta_{n+1}, \lambda\right) & =0 .
\end{aligned}
$$

Then, we can write

$$
T\left(\beta_{*}, \psi \beta_{*}, \lambda\right) \leq T\left(\beta_{*}, \beta_{n+1}, \frac{\lambda}{2}\right) \Delta T\left(\beta_{n+1}, \psi \beta_{*}, \frac{\lambda}{2}\right) .
$$

Taking limit as $n \longrightarrow+\infty$, we get

$$
T\left(\beta_{*}, \psi \beta_{*}, \lambda\right)=0 \Delta 0=0,
$$

so $\psi \beta_{*}=\beta_{*}$. Next proof is similar as in Theorem 1 .

Proof. of Theorem 2. Since $(E, R, S, T, *, \Delta, \perp)$ is an O-complete NMS, there exists $\beta_{0} \in E$, such that

$$
\begin{aligned}
& \beta_{0} \perp \delta, \\
& \forall \delta \in E .
\end{aligned}
$$

Thus, $\beta_{0} \perp \psi$. Consider

$$
\beta_{n}=\psi^{n} \beta_{0}=\psi \beta_{n-1}, \quad \forall n \in S .
$$

Since $\psi$ is $\perp$-preserving, $\left\{\beta_{n}\right\}$ is an $\mathrm{O}$-sequence. We can get

$$
\begin{aligned}
R\left(\beta_{n+1}, \beta_{n}, \lambda\right) & \geq R\left(\beta_{n+1}, \beta_{n}, \theta \lambda\right)=R\left(\psi \beta_{n}, \psi \beta_{n-1}, \theta \lambda\right) \\
& \geq \min \left\{R\left(\psi \beta_{n}, \beta_{n}, \lambda\right), R\left(\psi \beta_{n-1}, \beta_{n-1}, \lambda\right)\right\}, \\
S\left(\beta_{n+1}, \beta_{n}, \lambda\right) & \leq S\left(\beta_{n+1}, \beta_{n}, \theta \lambda\right)=S\left(\psi \beta_{n}, \psi \beta_{n-1}, \theta \lambda\right) \\
& \leq \min \left\{S\left(\psi \beta_{n}, \beta_{n}, \lambda\right), S\left(\psi \beta_{n-1}, \beta_{n-1}, \lambda\right)\right\}, \\
T\left(\beta_{n+1}, \beta_{n}, \lambda\right) & \leq T\left(\beta_{n+1}, \beta_{n}, \theta \lambda\right)=T\left(\psi \beta_{n}, \psi \beta_{n-1}, \theta \lambda\right) \\
& \leq \min \left\{T\left(\psi \beta_{n}, \beta_{n}, \lambda\right), T\left(\psi \beta_{n-1}, \beta_{n-1}, \lambda\right)\right\} .
\end{aligned}
$$

Two cases arise.

Case 1: if $R\left(\beta_{n+1}, \beta_{n}, \lambda\right) \geq R\left(\psi \beta_{n}, \beta_{n}, \lambda\right)$, then

$$
\begin{aligned}
R\left(\beta_{n+1}, \beta_{n}, \lambda\right) & \geq R\left(\beta_{n+1}, \beta_{n}, \theta \lambda\right) \\
& \geq R\left(\psi \beta_{n}, \beta_{n}, \lambda\right)=R\left(\beta_{n+1}, \beta_{n}, \lambda\right) \\
S\left(\beta_{n+1}, \beta_{n}, \lambda\right) & \leq S\left(\psi \beta_{n}, \beta_{n}, \lambda\right) .
\end{aligned}
$$

Then, 


$$
\begin{aligned}
S\left(\beta_{n+1}, \beta_{n}, \lambda\right) & \leq S\left(\beta_{n+1}, \beta_{n}, \theta \lambda\right) \\
& \leq S\left(\psi \beta_{n}, \beta_{n}, \lambda\right)=S\left(\beta_{n+1}, \beta_{n}, \lambda\right) \\
T\left(\beta_{n+1}, \beta_{n}, \lambda\right) & \leq T\left(\psi \beta_{n}, \beta_{n}, \lambda\right) .
\end{aligned}
$$

Then,

$$
\begin{aligned}
T\left(\beta_{n+1}, \beta_{n}, \lambda\right) & \leq T\left(\beta_{n+1}, \beta_{n}, \theta \lambda\right) \\
& \leq T\left(\psi \beta_{n}, \beta_{n}, \lambda\right)=T\left(\beta_{n+1}, \beta_{n}, \lambda\right) .
\end{aligned}
$$

Then by Lemma $1, \beta_{n}=\beta_{n+1}$ for all $n \in \mathbb{N}$.

Case 2: if $R\left(\beta_{n+1}, \beta_{n}, \lambda\right) \geq R\left(\psi \beta_{n-1}, \beta_{n-1}, \lambda\right)$, then

$$
\begin{aligned}
R\left(\beta_{n+1}, \beta_{n}, \lambda\right) & \geq R\left(\beta_{n+1}, \beta_{n}, \theta \lambda\right) \\
& \geq R\left(\psi \beta_{n-1}, \beta_{n-1}, \lambda\right) \geq R\left(\beta_{n}, \beta_{n-1}, \lambda\right), \\
S\left(\beta_{n+1}, \beta_{n}, \lambda\right) & \leq S\left(\psi \beta_{n-1}, \beta_{n-1}, \lambda\right) .
\end{aligned}
$$

Then,

$$
\begin{aligned}
S\left(\beta_{n+1}, \beta_{n}, \lambda\right) & \leq S\left(\beta_{n+1}, \beta_{n}, \theta \lambda\right) \\
& \leq S\left(\psi \beta_{n-1}, \beta_{n-1}, \lambda\right) \leq S\left(\beta_{n}, \beta_{n-1}, \lambda\right) \\
T\left(\beta_{n+1}, \beta_{n}, \lambda\right) & \leq T\left(\psi \beta_{n-1}, \beta_{n-1}, \lambda\right) .
\end{aligned}
$$

Then,

$$
\begin{aligned}
T\left(\beta_{n+1}, \beta_{n}, \lambda\right) & \leq T\left(\beta_{n+1}, \beta_{n}, \theta \lambda\right) \leq T\left(\psi \beta_{n-1}, \beta_{n-1}, \lambda\right) \\
& \leq T\left(\beta_{n}, \beta_{n-1}, \lambda\right),
\end{aligned}
$$

for all $n \in \mathbb{N}$ and $\lambda>0$. Then by Theorem 1 , we have a Cauchy O-sequence. By completeness of $(E, R, S, T, *, \Delta, \perp)$, there exists $\beta_{*} \in E$, such that

$$
\begin{aligned}
& \lim _{n \longrightarrow \infty} R\left(\beta_{n}, \beta_{*}, \lambda\right)=1, \\
& \lim _{n \longrightarrow \infty} S\left(\beta_{n}, \beta_{*}, \lambda\right)=0, \\
& \lim _{n \longrightarrow \infty} T\left(\beta_{n}, \beta_{*}, \lambda\right)=0, \\
& \text { for all } \lambda>0 .
\end{aligned}
$$

We know that $\psi$ is an $\perp$-continuous mapping, then

$$
\begin{aligned}
& \lim _{n \longrightarrow \infty} R\left(\beta_{n+1}, \psi \beta_{*}, \lambda\right)=\lim _{n \longrightarrow \infty} R\left(\psi \beta_{n}, \psi \beta_{*}, \lambda\right)=1, \\
& \lim _{n \longrightarrow \infty} S\left(\beta_{n+1}, \psi \beta_{*}, \lambda\right)=\lim _{n \longrightarrow \infty} S\left(\psi \beta_{n}, \psi \beta_{*}, \lambda\right)=0, \\
& \lim _{n \longrightarrow \infty} T\left(\beta_{n+1}, \psi \beta_{*}, \lambda\right)=\lim _{n \longrightarrow \infty} T\left(\psi \beta_{n}, \psi \beta_{*}, \lambda\right)=0 .
\end{aligned}
$$

Now, we prove that $\beta_{*}$ is a FP of $\psi$. Let $\lambda_{1} \in(\theta, 1)$ and $\lambda_{2}=1-\lambda_{1}$. Then,

$$
\begin{aligned}
R\left(\psi \beta_{*}, \beta_{*}, \lambda\right) \geq & R\left(\psi \beta_{*}, \beta_{n+1}, \frac{\lambda \lambda_{1}}{2}\right) * R\left(\beta_{n+1}, \beta_{*}, \frac{\lambda \lambda_{2}}{2}\right) \\
= & R\left(\psi \beta_{*}, \psi \beta_{n}, \frac{\lambda \lambda_{1}}{2}\right) * R\left(\beta_{n+1}, \beta_{*}, \frac{\lambda \lambda_{2}}{2}\right) \\
\geq & \min \left\{R\left(\psi \beta_{*}, \beta_{*}, \frac{\lambda \lambda_{1}}{2 \theta}\right), R\left(\psi \beta_{n}, \beta_{n}, \frac{\lambda \lambda_{1}}{2 \theta}\right)\right\} \\
& * R\left(\beta_{n+1}, \beta_{*}, \frac{\lambda \lambda_{2}}{2}\right) \\
= & \min \left\{R\left(\psi \beta_{*}, \beta_{*}, \frac{\lambda \lambda_{1}}{2 \theta}\right), R\left(\beta_{n+1}, \beta_{n}, \frac{\lambda \lambda_{1}}{2 \theta}\right)\right\} * R\left(\beta_{n+1}, \beta_{*}, \frac{\lambda \lambda_{2}}{2}\right) .
\end{aligned}
$$

Taking $n \longrightarrow \infty$, we get 


$$
\begin{aligned}
R\left(\psi \beta_{*}, \beta_{*}, \lambda\right) & \geq \min \left\{R\left(\psi \beta_{*}, \beta_{*}, \frac{\lambda \lambda_{1}}{2 \theta}\right), 1\right\} * 1, \\
R\left(\psi \beta_{*}, \beta_{*}, \lambda\right) & \geq R\left(\psi \beta_{*}, \beta_{*}, \frac{\lambda}{v}\right), \quad \lambda>0, \\
S\left(\psi \beta_{*}, \beta_{*}, \lambda\right) & \leq S\left(\psi \beta_{*}, \beta_{n+1}, \frac{\lambda \lambda_{1}}{2}\right) \Delta S\left(\beta_{n+1}, \beta_{*}, \frac{\lambda \lambda_{2}}{2}\right) \\
& =S\left(\psi \beta_{*}, \psi \beta_{n}, \frac{\lambda \lambda_{1}}{2}\right) \Delta S\left(\beta_{n+1}, \beta_{*}, \frac{\lambda \lambda_{2}}{2}\right) \\
& \leq \min \left\{S\left(\psi \beta_{*}, \beta_{*}, \frac{\lambda \lambda_{1}}{2 \theta}\right), S\left(\psi \beta_{n}, \beta_{n}, \frac{\lambda \lambda_{1}}{2 \theta}\right)\right\} \Delta S\left(\beta_{n+1}, \beta_{*}, \frac{\lambda \lambda_{2}}{2}\right) \\
& =\min \left\{S\left(\psi \beta_{*}, \beta_{*}, \frac{\lambda \lambda_{1}}{2 \theta}\right), S\left(\beta_{n+1}, \beta_{n}, \frac{\lambda \lambda_{1}}{2 \theta}\right)\right\} \Delta S\left(\beta_{n+1}, \beta_{*}, \frac{\lambda \lambda_{2}}{2}\right) .
\end{aligned}
$$

Taking $n \longrightarrow \infty$, we get

$$
\begin{aligned}
& S\left(\psi \beta_{*}, \beta_{*}, \lambda\right) \leq \min \left\{S\left(\psi \beta_{*}, \beta_{*}, \frac{\lambda \lambda_{1}}{2 \theta}\right), 0\right\} \Delta 0, \\
& S\left(\psi \beta_{*}, \beta_{*}, \lambda\right) \leq S\left(\psi \beta_{*}, \beta_{*}, \frac{\lambda}{v}\right), \quad \lambda>0, \\
& T\left(\psi \beta_{*}, \beta_{*}, \lambda\right) \leq T\left(\psi \beta_{*}, \beta_{n+1}, \frac{\lambda \lambda_{1}}{2}\right) \Delta T\left(\beta_{n+1}, \beta_{*}, \frac{\lambda \lambda_{2}}{2}\right) \\
&=T\left(\psi \beta_{*}, \psi \beta_{n}, \frac{\lambda \lambda_{1}}{2}\right) \Delta T\left(\beta_{n+1}, \beta_{*}, \frac{\lambda \lambda_{2}}{2}\right) \\
& \leq \min \left\{T\left(\psi \beta_{*}, \beta_{*}, \frac{\lambda \lambda_{1}}{2 \theta}\right), T\left(\psi \beta_{n}, \beta_{n}, \frac{\lambda \lambda_{1}}{2 \theta}\right)\right\} \Delta T\left(\beta_{n+1}, \beta_{*}, \frac{\lambda \lambda_{2}}{2}\right) \\
&=\min \left\{T\left(\psi \beta_{*}, \beta_{*}, \frac{\lambda \lambda_{1}}{2 \theta}\right), T\left(\beta_{n+1}, \beta_{n}, \frac{\lambda \lambda_{1}}{2 \theta}\right)\right\} \Delta T\left(\beta_{n+1}, \beta_{*}, \frac{\lambda \lambda_{2}}{2}\right) . \\
& \beta_{0} \perp \beta_{*}, \\
& \beta_{0} \perp \delta_{*} .
\end{aligned}
$$

Taking $n \longrightarrow \infty$, we get

$T\left(\psi \beta_{*}, \beta_{*}, \lambda\right) \leq \min \left\{T\left(\psi \beta_{*}, \beta_{*}, \frac{\lambda \lambda_{1}}{2 \theta}\right), 0\right\} \Delta 0$,

$T\left(\psi \beta_{*}, \beta_{*}, \lambda\right) \leq T\left(\psi \beta_{*}, \beta_{*}, \frac{\lambda}{v}\right), \quad \lambda>0$.

Here, $v=\left(2 \theta / \lambda_{1}\right) \in(0,1)$; from Lemma 1 , we have $\psi \beta_{*}=\beta_{*}$.

Take $\beta_{*}$ and $\delta_{*}$ to be two different FPs of $\psi$ to prove the uniqueness. We have
Because $\psi$ is an $\perp$-preserving, so we can write

$$
\begin{aligned}
& \psi^{n} \beta_{0} \perp \psi^{n} \beta_{*}, \\
& \psi^{n} \beta_{0} \perp \psi^{n} \delta_{*},
\end{aligned}
$$

for all $n \in \mathbb{N}$.

We can write 


$$
\begin{aligned}
& R\left(\psi^{n} \beta_{0}, \psi^{n} \beta_{*}, \lambda\right) \geq R\left(\psi^{n} \beta_{0}, \psi^{n} \beta_{*}, \theta \lambda\right) \geq \min \left\{R\left(\psi^{n} \beta_{0}, \beta_{0}, \lambda\right), R\left(\psi^{n} \beta_{*}, \beta_{*}, \lambda\right)\right\}, \\
& R\left(\psi^{n} \beta_{0}, \psi^{n} \delta_{*}, \lambda\right) \geq R\left(\psi^{n} \beta_{0}, \psi^{n} \delta_{*}, \theta \lambda\right) \geq \min \left\{R\left(\psi^{n} \beta_{0}, \beta_{0}, \lambda\right), R\left(\psi^{n} \delta_{*}, \delta_{*}, \lambda\right)\right\} .
\end{aligned}
$$

Hence, we write that

$$
\begin{gathered}
R\left(\beta_{*}, \delta_{*}, \lambda\right)=R\left(\psi^{n} \beta_{*}, \psi^{n} \delta_{*}, \lambda\right) \geq \min \left\{R\left(\psi^{n} \beta_{*}, \beta_{*}, \frac{\lambda}{\theta}\right), R\left(\psi^{n} \delta_{*} \delta_{*}, \frac{\lambda}{\theta}\right)\right\}=\min \{1,1\}=1, \\
S\left(\psi^{n} \beta_{0}, \psi^{n} \beta_{*}, \lambda\right) \leq S\left(\psi^{n} \beta_{0}, \psi^{n} \beta_{*}, \theta \lambda\right) \leq \min \left\{S\left(\psi^{n} \beta_{0}, \beta_{0}, \lambda\right), S\left(\psi^{n} \beta_{*}, \beta_{*}, \lambda\right)\right\}, \\
S\left(\psi^{n} \beta_{0}, \psi^{n} \delta_{*}, \lambda\right) \leq S\left(\psi^{n} \beta_{0}, \psi^{n} \delta_{*}, \theta \lambda\right) \leq \min \left\{S\left(\psi^{n} \beta_{0}, \beta_{0}, \lambda\right), S\left(\psi^{n} \delta_{*}, \delta_{*}, \lambda\right)\right\} .
\end{gathered}
$$

Hence, we write that

$$
\begin{aligned}
S\left(\beta_{*}, \delta_{*}, \lambda\right) & =S\left(\psi^{n} \beta_{*}, \psi^{n} \delta_{*}, \lambda\right) \leq \min \left\{S\left(\psi^{n} \beta_{*}, \beta_{*}, \frac{\lambda}{\theta}\right), S\left(\psi^{n} \delta_{*}, \delta_{*}, \frac{\lambda}{\theta}\right)\right\}=\min \{0,0\}=0, \\
T\left(\psi^{n} \beta_{0}, \psi^{n} \beta_{*}, \lambda\right) & \leq T\left(\psi^{n} \beta_{0}, \psi^{n} \beta_{*}, \theta \lambda\right) \leq \min \left\{T\left(\psi^{n} \beta_{0}, \beta_{0}, \lambda\right), T\left(\psi^{n} \beta_{*}, \beta_{*}, \lambda\right)\right\}, \\
T\left(\psi^{n} \beta_{0}, \psi^{n} \delta_{*}, \lambda\right) & \leq T\left(\psi^{n} \beta_{0}, \psi^{n} \delta_{*}, \theta \lambda\right) \leq \min \left\{T\left(\psi^{n} \beta_{0}, \beta_{0}, \lambda\right), T\left(\psi^{n} \delta_{*}, \delta_{*}, \lambda\right)\right\} .
\end{aligned}
$$

Hence, we write that

$$
T\left(\beta_{*}, \delta_{*}, \lambda\right)=T\left(\psi^{n} \beta_{*}, \psi^{n} \delta_{*}, \lambda\right) \leq \min \left\{T\left(\psi^{n} \beta_{*}, \beta_{*}, \frac{\lambda}{\theta}\right), T\left(\psi^{n} \delta_{*}, \delta_{*}, \frac{\lambda}{\theta}\right)\right\}=\min \{0,0\}=0
$$

for all $\lambda>0$. Hence, $\beta_{*}=\delta_{*}$.

\section{Data Availability}

No data were used to support this study.

\section{Conflicts of Interest}

The authors declare that they have no conflicts of interest.

\section{Acknowledgments}

The authors are grateful to the Basque Government for supporting and funding this research article (IT1207-19).

\section{References}

[1] L. A. Zadeh, "Fuzzy sets," Information and Control, vol. 8, no. 3, pp. 338-353, 1965.

[2] Z. Deng, "Fuzzy pseudo-metric spaces," Journal of Mathematical Analysis and Applications, vol. 86, no. 1, pp. 74-95, 1982.

[3] O. Kaleva and S. Seikkala, "On fuzzy metric space," Fuzzy Sets and Systems, vol. 12, no. 3, pp. 215-229, 1984.
[4] K. Javed, F. Uddin, H. Aydi, A. Mukheimer, and M. Arshad, "Ordered-theoretic fixed point results in fuzzy b-metric spaces with an application," Journal of Mathematics, vol. 2021, Article ID 6663707, 2021.

[5] K. Javed, F. Uddin, H. Aydi, M. Arshad, U. Ishtiaq, and H. Alsamir, "On fuzzy b-metric-like spaces," Journal of Function Spaces, vol. 2021, Article ID 6615976, 2021.

[6] F. Uddin, K. Javed, H. Aydi, U. Ishtiaq, and M. Arshad, "Control fuzzy metric spaces via orthogonality with an application," Journal of Mathematics, vol. 2021, Article ID 5551833, 2021.

[7] R. Verma and B. D. Sharma, "Fuzzy generalized prioritized weighted average operator and its application to multiple attribute decision making," International Journal of Intelligent Systems, vol. 29, no. 1, pp. 26-49, 2014.

[8] J. Zhou, W. Pedrycz, X. Yue, C. Gao, Z. Lai, and J. Wan, "Projected fuzzy C-means clustering with locality preservation,” Pattern Recognition, vol. 113, 2021.

[9] R. Verma and S. Maheshwari, "A new measure of divergence with its application to multi-criteria decision making under fuzzy environment," Neural Computing and Applications, vol. 28 , no. 8, pp. 2335-2350, 2017.

[10] M. Hezarjaribi, "Fixed point result in orthogonal fuzzy metric space," Jordan Journal of Mathematics and Statistics (JJMS), vol. 11, no. 4, pp. 295-308, 2018. 
[11] J. H. Park, "Intuitionistic fuzzy metric spaces," Chaos, Solitons \& Fractals, vol. 22, no. 5, pp. 1039-1046, 2004.

[12] M. Kirișci and N. Simsek, "Neutrosophic metric spaces," Mathematical Sciences, vol. 14, pp. 241-248, 2020.

[13] N. Simsek and M. Kirişci, "Fixed point theorems in Neutrosophic metric spaces," Sigma Journal of Engineering and Natural Sciences, vol. 10, no. 2, pp. 221-230, 2019.

[14] S. Sowndrarajan, M. Jeyarama, and F. Smarandache, "Fixed point results for contraction theorems in neutrosophic metric spaces," Neutrosophic Sets and Systems, vol. 36, p. 1, 2020.

[15] M. Eshaghi, M. Ramezani, M. D. L. Sen, and Y. J. Cho, "On orthogonal sets and Banach's fixed point theorem," Fixed Point Theory, vol. 18, pp. 569-578, 2017.

[16] T. Senapati, L. K. Dey, B. Damjanović, and A. Chanda, "New fixed point results in orthogonal metric spaces with an application," Kragujevac Journal of Mathematics, vol. 42, no. 4, pp. 505-516, 2018.

[17] K. Javed, H. Aydi, F. Uddin, and M. Arshad, "On orthogonal partial b-metric spaces with an application," Journal of Mathematics, vol. 2021, Article ID 6692063, 2021.

[18] F. Uddin, C. Park, K. Javed, M. Arshad, and J. R. Lee, “Orthogonal $\mathrm{m}$-metric spaces and an application to solve integral equations," Advances in Difference Equations, vol. 159, 2021.

[19] F. Smarandache, A Unifying Field in Logics. Neutrosophy: Neutrosophic Probability, Set and Logic, American Research Press, Champaign, IL, USA, 1999. 